\title{
HsTRPA of the Red Imported Fire Ant, Solenopsis invicta, Functions as a Nocisensor and Uncovers the Evolutionary Plasticity of HsTRPA Channels
}

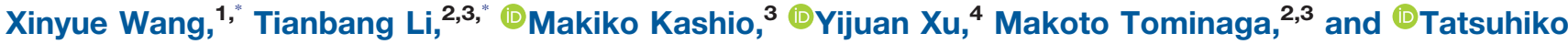 \\ Kadowaki ${ }^{1}$
}

DOI:http://dx.doi.org/10.1523/ENEURO.0327-17.2018

${ }^{1}$ Department of Biological Sciences, Xi'an Jiaotong-Liverpool University, Jiangsu Province 215123, China,

${ }^{2}$ Department of Physiological Sciences, SOKENDAl (the Graduate University for Advanced Studies), Okazaki, 4448585, Japan, ${ }^{3}$ Division of Cell Signaling, Okazaki Institute for Integrative Bioscience, National Institutes of Natural Sciences, Okazaki, 444-8787, Japan, ${ }^{4}$ Department of Entomology, South China Agricultural University, Guangzhou, Guangdong Province 510642, China

\section{Visual Abstract}
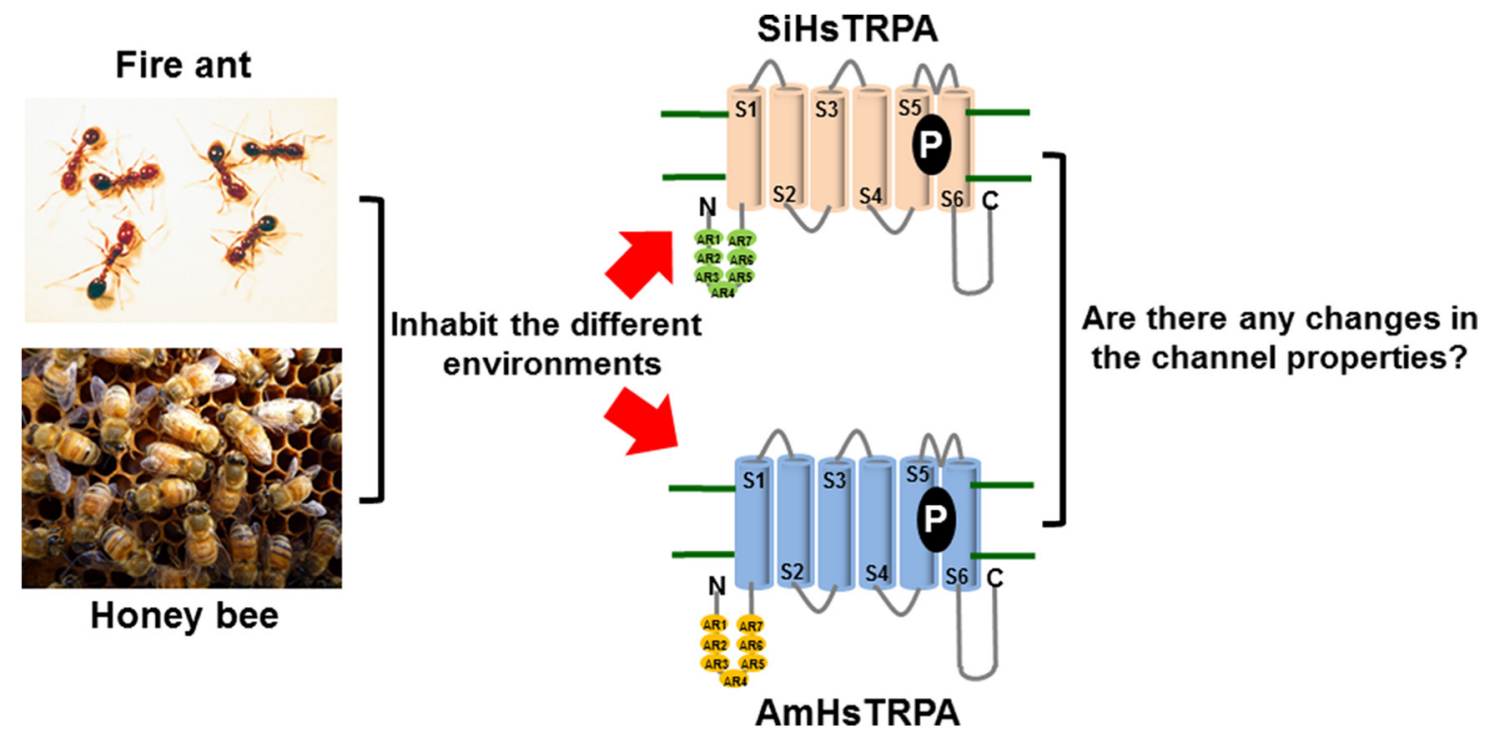

\section{Significance Statement}

TRP channels have crucial roles in the perception of various sensory stimuli by functioning as signal integrators. They have rapidly evolved at multiple levels, and hence the chemical and temperature activation profiles of orthologous TRP channels can differ even between closely related species. Two major questions remain to be answered: what are the physiological consequences of such changes in channel properties, and have these changes been driven by adaptive evolution? The comparison between HsTRPA (SiHsTRPA) of the red imported fire ant and the honey bee ortholog, AmHsTRPA, reported here gives insights into the above questions. Furthermore, SiHsTRPA may serve as a new molecular target in strategies to control the fire ant, a major invasive alien species. 
Solenopsis invicta, the red imported fire ant, represents one of the most devastating invasive species. To understand their sensory physiology, we identified and characterized their Hymenoptera-specific (Hs) TRPA channel, SiHsTRPA. Consistent with the sensory functions of SiHsTRPA, it is activated by heat, an electrophile, and an insect repellent. Nevertheless, SiHsTRPA does not respond to most of the honey bee ortholog (AmHsTRPA)-activating compounds. The jewel wasp ortholog (NvHsTRPA) is activated by these compounds even though it outgroups both AmHsTRPA and SiHsTRPA. Characterization of AmHsTRPA/SiHsTRPA chimeric channels revealed that the amino acids in the $\mathrm{N}$ terminus, as well as ankyrin repeat 2 (AR2) of AmHsTRPA, are essential for the response to camphor. Furthermore, amino acids in ARs 3 and 5-7 were specifically required for the response to diallyl disulfide. Thus, amino acid substitutions in the corresponding domains of SiHsTRPA during evolution would be responsible for the loss of chemical sensitivity. SiHsTRPA-activating compounds repel red imported fire ants, suggesting that SiHsTRPA functions as a sensor for noxious compounds. SiHsTRPA represents an example of the species-specific modulation of orthologous TRPA channel properties by amino acid substitutions in multiple domains, and SiHsTRPA-activating compounds could be used to develop a method for controlling red imported fire ants.

Key words: Evolutionary plasticity; fire ant; honey bee; HsTRPA channel; sensor for noxious stimuli

\section{Introduction}

Transient receptor potential (TRP) channels are different from most other cation channels, as they display diverse cation selectivity and activation mechanisms (Venkatachalam and Montell, 2007; Julius, 2013). TRP channels function in various sensory perception pathways such as vision, thermosensation, olfaction, hearing, taste sensation, and mechanosensation (Damann et al., 2008). Individual cells use TRP channels to detect local changes in temperature, osmolarity, and fluid flow in their environment (Venkatachalam and Montell, 2007; Nilius and Owsianik, 2011). TRP channels are evolutionarily plastic and dynamic at multiple levels: gene gain/loss, amino acid substitutions, transcription, and pre-mRNA splicing (Kadowaki, 2015). Thus, TRP channels have undergone more rapid evolution than several conserved cation channels,

Received September 18, 2017; accepted January 18, 2018; First published February 5, 2018

Authors report no conflict of interest.

Author Contributions: XW, TL, and MK conducted the experiments and YX collected the red imported fire ants. TK designed and supervised the experiments and wrote the manuscript. MT supervised the experiments and helped revision of manuscript together with MK.

This work was supported by 2012 Suzhou Science and Technology Development Planning Programme (Grant\#: SYN201213) and Jinji Lake Double Hundred Talents Programme to TK.

${ }^{*} \mathrm{X} . \mathrm{W}$. and T.L. contributed equally to this work.

Makiko Kashio's present address is Department of Physiology, Aichi Medical University, Nagakute 480-1195, Japan.

Acknowledgments: We thank Craig Montell, Paul Garrity, Teiichi Tanimura, and Bloomington Stock Center for providing us fruit fly stocks for the experiments. We are grateful to Xiao Shi, Qing Zhang, Yixuan Lin, Sijing Wang, Chang Liu, Tiange Li, Li Shen, Hanshuo Lu, Jing He, Weile Chen, Ziyue Wang, Tianyang Yu, and Wen Wang for participating in some of the experiments. We also thank C. David O'Connor for helping us to revise the Significance Statement and Ziwen Xie for preparing the fruit fly food.

Correspondence should be addressed to Tatsuhiko Kadowaki, Department of Biological Sciences, Xi'an Jiaotong-Liverpool University, 111 Ren'ai Road, Suzhou Dushu Lake Higher Education Town, Jiangsu Province 215123, China. E-mail: Tatsuhiko.Kadowaki@xjtlu.edu.cn.

DOI:http://dx.doi.org/10.1523/ENEURO.0327-17.2018

Copyright (C) 2018 Wang et al.

This is an open-access article distributed under the terms of the Creative Commons Attribution 4.0 International license, which permits unrestricted use, distribution and reproduction in any medium provided that the original work is properly attributed. such as $\mathrm{K}^{+}$channels (Kadowaki, 2015; Saito and Tominaga, 2015).

The metazoan TRP superfamily is classified into eight subfamilies-TRPA, TRPC, TRPM, TRPML, TRPN, TRPP, TRPV, and TRPVL-according to a phylogenetic tree based on the amino acid sequences of six transmembrane segments (Peng et al., 2015a). Among them, the TRPA subfamily contains ankyrin repeats (ARs) in the $N$ terminus. ARs are 33-residue motifs consisting of pairs of antiparallel $\alpha$-helices connected by $\beta$-hairpin motifs and have important roles in the chemical and temperature reactivity of TRPA1 channels (Hinman et al., 2006; Macpherson et al., 2007; Cordero-Morales et al., 2011; Jabba et al., 2014). Among TRPA subfamily members, TRPA1 channels have been best characterized; however, other insect- and crustacean-specific TRPA channels, Painless (Pain), Pyrexia (Pyx), Waterwitch (Wtrw), and HsTRPA, have also been characterized (Tracey et al., 2003; Lee et al., 2005; Liu et al., 2007; Sokabe et al., 2008; Matsuura et al., 2009; Sun et al., 2009; Kohno et al., 2010; Wolfgang et al., 2013). All channels except Wtrw were shown to be heat sensitive, and honey bee HsTRPA (AmHsTRPA) is also activated by electrophiles and camphor (Kohno et al., 2010). Because the ancient nocisensor, TRPA1, is absent in the genome-sequenced Hymenoptera, it was proposed that AmHsTRPA was generated by duplication of honey bee Wtrw and has undergone neofunctionalization to gain heat and chemical sensitivity to complement the loss of TRPA1 function in honey bees (Kohno et al., 2010). Heat activation of jewel wasp (Nasonia vitripennis) HsTRPA (NvHsTRPA) was also briefly reported (Matsuura et al., 2009). Evolutionary plasticity was also well documented within TRPA1 channels (Kadowaki, 2015; Saito and Tominaga, 2015). Although gene gain/loss events in other TRPA subfamily members were previously reported (Peng et al., 2015a), plasticity by amino acid substitutions has not yet been shown with any member.

The red imported fire ant (RIFA), Solenopsis invicta, is one of the major invasive alien species in the United States, China, Australia, and other regions of the world. The invasion of $S$. invicta not only represents threats to the local ecosystem (Morrison, 2002; Wilder et al., 2013), 
but also affects agricultural production (Wu et al., 2014). In addition, S. invicta causes public health concerns because of the potential serious clinical symptoms of stings (Fernandez-Melendez et al., 2007). Owing to the variety of negative impacts of invasive RIFA (Vinson, 2013), a number of methods to manage RIFAs have been developed, and baits and conventional contact insecticides such as chlorpyrifos and pyrethroids have been widely used. However, the use of these chemicals often creates public health and environmental problems. To avoid these issues, it is important to develop new strategies to control RIFAs. Therefore, identifying safe and natural insecticides/repellents has gotten a lot of attention, and several reports have shown that some plant-derived compounds, particularly from essential oils, effectively repel and/or kill RIFAs (Wheeler et al., 2003; Appel et al., 2004; Chen et al., 2008; Kafle and Shih, 2013; Zhang et al., 2014). However, the mechanisms of how these compounds repel RIFAs have not yet been revealed.

To investigate the functions and evolution of insect and crustacean-specific TRPA subfamily members in insects other than the fruit fly, Drosophila melanogaster, and identify potential compounds to repel RIFAs, we identified S. invicta HsTRPA (SiHsTRPA) and characterized its physiological functions in comparison with AmHsTRPA. Ants represent a major component of most terrestrial ecosystems, and many species show an enormous diversity in life history traits, ecological and behavioral adaptations, and social organization. Sensory systems of ants must play important roles for the above habitats and behaviors; nevertheless, these systems still remain poorly understood. Characterization of SiHsTRPA should give insight into the roles of TRP channels in the ant sensory system. Additionally, we discuss the evolutionary plasticity of HsTRPA by comparing the properties of SiHsTRPA and AmHsTRPA channels.

\section{Materials and Methods}

\section{$5^{\prime}$ and $3^{\prime}$ RACE of SiHsTRPA}

To identify SiHsTRPA, we blast searched for S. invicta genomic scaffolds (Sinv 1.0 Scaffold Assembly) and the predicted transcripts (Sinv_OGSv2.2.3_cds) using AmHsTRPA sequence (accession no. LC322996) as a query (Wurm et al., 2011; Elsik et al., 2016). Two sequences similar to AmHsTRPA corresponded to SiHsTRPA and Siwtrw. To identify $5^{\prime}$ and $3^{\prime}$ ends of SiHsTRPA, S. invicta total RNA and two primers, 5' -GCGGATAATCCTTCGCTGC ATAAGTGC-3' (for the first PCR) and 5'-AATGG ATAGCGGATCGCGTTTGTGACG-3' (for the second PCR) were used for 5' RACE with 5'-Full RACE Kit (Takara). 3' RACE was conducted as above using two primers, $5^{\prime}$ CCCTCGTCTGCGAATGACGACATCAAT-3' (for the first PCR) and 5'-GCGATCACGCCGTTTGGCTGAAACATC-3' (for the second PCR) with SMARTer RACE cDNA Amplification Kit (Clontech Laboratories).

\section{Construction of SiHsTRPA-expressing vector for mammalian cells}

We isolated full-length SiHsTRPA cDNA by RT-PCR using S. invicta total RNA and the following two primers;
5'-TGAATTCACCATGTGTGAGAATGATAAAGATTCGTTA GG-3' and 5'-AATTTGCGGCCGCCTAATGAGGTATITCTTCTITCGCCAATGT-3'. The PCR product was digested with EcoRI and Notl, and then cloned in pAc5.1/ V5-His B vector (Thermo Scientific) in which D. melanogaster actin $5 \mathrm{C}$ promoter was replaced with CMV promoter. The SiHsTRPA protein expressed by this construct was tagged with a V5-epitope at the $C$ terminus, and this was used for verifying the expression and the cellular localization in HEK 293 cells (ATCC PTA-5077, RRID:CVCL_6911) by Western blot and immunofluorescence with rabbit anti-V5-epitope antibody (Sigma-Aldrich V8137, RRID:AB_26188), respectively. The staining pattern of V5-epitope-tagged SiHsTRPA channel was compared with FITC-WGA, which specifically labels the plasma membrane (Chazotte, 2011). A construct expressing untagged SiHsTRPA protein was then prepared using the above DNA construct as a template, the above primer with initiation codon, and the primer $5^{\prime}$-AATTT GCGGCCGCCCTATAATGAGGTATTTTCTTCTTTCGCCAA-3'. This DNA construct was used for all of the experiments described in the text.

\section{Construction of SiHsTRPA and AmHsTRPA mutants as well as AmHsTRPA/SiHsTRPA chimeric channels}

$\mathrm{N}$-terminal deletion (amino acid 21-98) mutant of AmHsTRPA was constructed by overlap extension PCR (Lee et al., 2010). Two PCR products were first prepared using the following primers; 5'-AATTAACCCTCACTAAA GGG-3' (T3) and 5'-ATCCGTGTAAAGTTGCAAGCTAGCCTT-3' (P2; upstream fragment) and 5'-TTGC AACTTTACACGGATGAAGACAGA-3' (P3) and 5'CGATCAACTGCGTTGACATTG-3' (P4; downstream fragment) and AmHsTRPA expression vector (Kohno et al., 2010) as a template. The second PCR was performed by mixing above two PCR products and using T3 and $\mathrm{P} 4$ as the primers, and then the resulting PCR product was digested with Notl and Xbal followed by replacing the corresponding fragment in the AmHsTRPA expression vector. AmHsTRPA $\mathrm{N}$-terminal insertion (amino acid 1-98) to SiHsTRPA was also constructed as above. The upstream fragment was prepared using primers, 5'-TTTAAGAATTCA CCATGGACGATAAACAGATA-3' (P5) and 5' CTCACACATCAGGCAGGAACGACGATT-3' (P6) and the AmHsTRPA expression vector as a template. The downstream fragment was prepared using primers, 5'TCCTGCCTGATGTGTGAGAATGATAAA-3' (P7) and 5'TCTCTCGCTITTGCACGCGTT-3' (P8) and the SiHsTRPA expression vector as a template. The second PCR was performed by mixing above two PCR products and using P5 and $\mathrm{P} 8$ as the primers, and then the resulting PCR product was digested with EcoRI and Sphl followed by replacing the corresponding fragment in the SiHsTRPA expression vector.

All of the AmHsTRPA/SiHsTRPA chimeric channels were constructed by overlap extension second PCR of the two or three first PCR amplicons. Both upstream and downstream fragments were PCR amplified using the AmHsTRPA expression vector as a template and middle fragment was PCR amplified using the SiHsTRPA expression vector as a template. The $\mathrm{N}$ terminus swapped AmHsTRPA chimera was constructed using three ampli- 
cons; the upstream fragment prepared by $\mathrm{T} 3$ and $5^{\prime}$ CGAATCTITGGTCAAGAAATTTCTGTC-3' (P9) primers, the downstream fragment prepared by $5^{\prime}$-GAGAGA CTAAGAAATATAGCGTACATG-3' (P10) and P4 primers, and the middle fragment prepared by $5^{\prime}$-TTCTT GACCAAAGATTCGTTAGGAGAC-3' (P11) and 5'TATATITCTTAGTCTCTCGCTITTGCA-3' (P12). The AR1swapped AmHsTRPA was constructed using three amplicons; the upstream fragment prepared by $\mathrm{T} 3$ and $5^{\prime}$ GTTCCGTAGTCGTTCTCCTGCCGGTAT-3' (P13) primers, the downstream fragment prepared by $5^{\prime}$-GAACC GCGAACGGGCATGAACGCGATC-3' (P14) and P4 primers, and the middle fragment prepared by $5^{\prime}$-GGAGA ACGACTACGGAACGTCGCTTAC-3' (P15) and 5' CATGCCCGTTCGCGGTTCGACGTAGTT-3' (P16). The AR2-swapped AmHsTRPA was constructed using three amplicons; the upstream fragment prepared by $\mathrm{T} 3$ and $5^{\prime}-$ CACGCCGGTGCTAGGCTCCACGTAATC-3' (P17) primers, the downstream fragment prepared by 5'-GAG AGCCTCATCAATCATTACACGCCT-3' (P18) and P4 primers, and the middle fragment prepared by $5^{\prime}-$ GAGCCTAGCACCGGCGTGAACGCTATC-3' (P19) and 5'ATGATTGATGAGGCTCTCATAATTTAC-3' (P20). The AR3swapped AmHsTRPA was constructed using three amplicons; the upstream fragment prepared by $\mathrm{T} 3$ and $5^{\prime}-$ GTAATGATTGATGAGGCTCTCATAATT-3' (P21) primers, the downstream fragment prepared by $5^{\prime}-\mathrm{TAC}$ GTCTGTCAGGAGGTGGCCGAACCA-3' (P22) and P4 primers, and the middle fragment prepared by $5^{\prime}$-AATC CGATCACTCGTTACACACCGCTT-3' (P23) and 5' CACCTCCTGACAGACGTAATTGAGCTT-3' (P24). The second PCR for above chimeras was performed using T3 and P4 as the primers, and the amplicon was processed as above for $\mathrm{N}$-terminal-deletion AmHsTRPA mutant. The AR3-5 swapped AmHsTRPA was constructed using three amplicons; the upstream fragment prepared by $\mathrm{T} 3$ and $5^{\prime}-$ GTAACGAGTGATCGGATTCCGGTAATT-3' (P25) primers, the downstream fragment prepared by $5^{\prime}-\mathrm{GTC}$ GACAGGGCACACAGAACCCCACTT-3' (P26) and 5'GCCTCGTCGTGGTTGTAACA-3' (P27) primers, and the middle fragment prepared by $5^{\prime}$-AATCCGATCACTC GTTACACACCGCTT-3' (P28) and 5' -TCTGTGT GCCCTGTCGACGGCGTTCAC-3' (P29). The second PCR was performed using T3 and P27 as the primers, and then the amplicon was digested with Notl and EcoRI followed by replacing the corresponding fragment in the AmHsTRPA expression vector. The AR5-7 swapped AmHsTRPA was constructed as above except using the following three amplicons; the upstream fragment prepared by $\mathrm{T} 3$ and $5^{\prime}-$ CTGGCCCAGATGGTIITCTCGACGAC-3' (P30) primers, the downstream fragment prepared by 5'-GTCGA CAGGGCACACAGAACCCCACTT-3' (P31) and P27 primers, and the middle fragment prepared by $5^{\prime}$ AAAAACCATCTGGGCCAGACGCCGTTG-3' (P32) and 5'AAGTCCCGCGCGCGTGGTCGCACTGAT-3' (P33). The boundary between ARs and transmembrane segments swapped AmHsTRPA was constructed using three amplicons; the upstream fragment prepared by $5^{\prime}$-TCAT GTGGTCGATCGTCACT-3' (P34) and 5'-GCCGGTGC GGGTGGTCGCACTGACGTCCGCACCGGCCCAAATTA- $3^{\prime}$ (P35) primers, the downstream fragment prepared by 5'-CAGGAGCACCGTAAAGATTTGCTCTCC-3' (P36) and P27 primers, and the middle fragment prepared by $5^{\prime}$ GCGACCACCCGCACCGGCTTGTCTGCT-3' (P37) and 5'ATCTITACGGTGCTCCTGTACGAAAGT-3' (P38). The second PCR was performed using P34 and P27 as the primers, and then the amplicon was digested with Xbal and EcoRI followed by replacing the corresponding fragment in the AmHsTRPA expression vector. The selectivity filter swapped AmHsTRPA was constructed using three amplicons; the upstream fragment prepared by $5^{\prime}$-CCGTT GGTAATGGCTTTCCT-3' (P39) and 5'-CAATTCA CCGGCCATCATTGCCAATAC-3' (P40) primers, the downstream fragment prepared by 5 '-CTCTTCACGCTGTT CATCGTGTTTGTT-3' (P41) and 5'-AAGGCTCTCAAG GGCATCGGT-3' (P42) primers, and the middle fragment prepared by 5'-ATGATGGCCGGTGAATTGGATTTCGAA-3' (P43) and 5'-GATGAACAGCGTGAAGAGGATCTGCGA-3' (P44). The second PCR was performed using P39 and P42 as the primers, and then the amplicon was digested with BamHI and EcoRI followed by replacing the corresponding fragment in the AmHsTRPA expression vector. The $\mathrm{C}$ terminus swapped AmHsTRPA was constructed using two amplicons; the upstream fragment prepared by P39 and 5'CGCAGACGACGGTATATIITCTGTGC-3' (P45) primers and the downstream fragment prepared by 5'AATATACCGTCGTCTGCGAATGACGAC-3' (P46) and P42 primers. The second PCR followed by the restriction enzyme digestion were the same as above.

\section{RT-PCR and qRT-PCR}

Total RNA was isolated from the abdomen, antennae, heads, legs, and thoraxes of 20 fire ants using Trizol reagent (Thermo Fisher Scientific). Total RNA was reverse transcribed using random primer and ReverTra Ace reverse transcriptase (Toyobo). The RT products were then used for PCR with the following primers; 5'-TAACTGG CGTGATGTGGGAC-3' ${ }^{\prime}$ and 5'-CATGCACGCAGTATTCA GGC-3' (for S. invicta 18S rRNA), 5' -AGGTCTGAGGAAA CACGCAG-3' and 5'-TCGGAAGGCATACGGGATTG-3' (for SiHsTRPA). The PCR was repeated for 25 and 35 cycles for $18 S$ rRNA and SiHsTRPA, respectively. The resulting PCR products were sequenced to verify their identities. The same primer sets and SYBR Green were used for qRT-PCR, and total RNA samples were prepared from three batches of collected tissues.

\section{$\mathrm{Ca}^{2+}$-imaging method (HEK293 cells)}

For $\mathrm{Ca}^{2+}$-imaging, $1 \mu \mathrm{g}$ SiHsTRPA expression vector and $0.1 \mu \mathrm{g}$ pCMV-DsRed expression vector were transfected to HEK293 cells in OPTI-MEM medium (Thermo Fisher Scientific) using Lipofectamine Plus reagents (Thermo Fisher Scientific). After incubating for 3-4 h at $37^{\circ} \mathrm{C}$, cells were reseeded on cover glasses and further incubated at $33^{\circ} \mathrm{C}$. The cells were used for the experiments 20-40 h after transfection. Transfected HEK293 cells on a cover glass were incubated in culture medium containing $5 \mu \mathrm{M}$ fura-2 AM (Thermo Fisher Scientific) at $33^{\circ} \mathrm{C}$ for $1-2 \mathrm{~h}$. The cover glass was washed, and fura-2 fluorescence was measured in a standard bath solution containing (in mM) $140 \mathrm{NaCl}, 5 \mathrm{KCl}, 2 \mathrm{MgCl}_{2}, 2 \mathrm{CaCl}_{2}, 10$ 
Hepes, and 10 glucose, pH 7.4 adjusted with $\mathrm{NaOH}$. A cover glass was mounted in a chamber (RC-26G, Warner Instruments) connected to a gravity flow system to deliver hot or cold bath solution for temperature stimulations and bath solution containing the tested compounds. The concentration of each compound was $1 \mathrm{~mm}$ except for carvacrol, geranylacetone, nerol $(0.5 \mathrm{~mm})$, menthol $(3 \mathrm{~mm})$, camphor $(5 \mathrm{~mm})$, saturated fatty acid $(0.2 \mathrm{~mm})$, and creosote $(0.1 \%)$. The emitted fluorescence $(510 \mathrm{~nm})$ by 340 and $380 \mathrm{~nm}$ were measured by CCD camera (CoolSNAP ES, Roper Scientific Photometrics), and the Fura-2 ratios $(340 / 380 \mathrm{~nm})$ were acquired and recorded every $3 \mathrm{~s}$ by IPlab software (Scanalytics). Changes of Fura-2 ratio over time were analyzed by ImageJ. Each recording was repeated twice except for stimulation of SiHsTRPA by heat or $\beta$-citronellol and stimulation of AmHsTRPA by octanoic acid (three times each). To assess activation of the channel by the specific stimulation, we calculated the fold change of Fura-2 ratio (the maximum Fura-2 ratio recorded after the stimulation divided by Fura-2 ratio just before the stimulation) in the individual DsRed-positive (with the channel expression) and DsRed-negative (without the channel expression) cells, and then statistically compared the values.

\section{Genetics}

UAS-SiHsTRPA fruit fly was generated by integrating the transgene to $68 \mathrm{~A} 4$ by PhiC31 integrase-mediated recombination event (Bischof et al., 2007). The transgene was driven by Gr33a-Gal4 (Moon et al., 2009). The above transgene was also recombined with 20XUAS-IVS-GCaMP6m (Chen et al., 2013) on the third chromosome, and then their expression was driven by Gr64f-Gal4 (Jiao et al., 2008) with two copies for each UAS and Ga/4 transgene.

\section{Assay for thermotactic behavior of $D$. melanogaster}

To assay the temperature preference of fruit flies, a temperature gradient of $14-37^{\circ} \mathrm{C}$ with a slope of $0.94^{\circ} \mathrm{C} / \mathrm{cm}$ was produced in an aluminum block ( 27 long $\times 15$ wide $\times$ $2.5 \mathrm{~cm}$ high) as previously reported (Sayeed and Benzer, 1996). Thermometers were embedded in the block every $2.4 \mathrm{~cm}$, and the gradient was established using a cold circulating water chamber and a hot probe at each end. The aluminum block was covered with moist paper to maintain a uniform relative humidity along the gradient. This paper was divided into 20 observation fields with a black pencil for recording the distribution of fruit flies. A glass plate with three separate lanes was placed $5 \mathrm{~mm}$ above the block, creating suitable corridors for fruit flies to migrate. Forty to fifty fruit flies per lane were placed in the middle of the testing arena around $25^{\circ} \mathrm{C}$ between the aluminum block and the glass plate, allowed to migrate for $3 \mathrm{~h}$, and photographed every $10 \mathrm{~min}$ with a digital camera. Fruit flies moved very rapidly in the entire area when they were first introduced into the apparatus; however, they gradually ceased rapid movement in the apparatus. We then counted the number of fruit flies in each observation field and divided by the total number of fruit flies in the entire area every 10 min between 1.5 and $2.5 \mathrm{~h}$ after the introduction. The average value of six recordings was calculated for each experimental group. Before intro- ducing fruit flies into the temperature gradient apparatus, they were placed in plastic vials and cooled on ice until they stopped moving. All experiments were performed in a room where the temperature was kept constant at $25^{\circ} \mathrm{C}$.

\section{Assay for thermal nociception behavior of $D$. melanogaster larvae}

To test the thermal nociception behavior of $D$. melanogaster larvae, the actively wandering third instar larvae were collected from vials and transferred to $35-\mathrm{mm}$ plastic Petri dishes with water to support exhibiting the full rolling response. Larvae were touched laterally in abdominal segments (A4-A6) with the hot probe. In all experiments, 20-40 larvae were tested per group in four replicates. The behavioral responses were recorded and analyzed as described (Tracey et al., 2003; Neely et al., 2011; Zhong et al., 2012). To calculate the median of response latency of each genotype, the latency of larvae responding over $10 \mathrm{~s}$ was considered as 10 , although some of the larvae never responded to touching by the hot probe.

\section{Proboscis extension response assay of $D$. melanogaster}

The proboscis extension response (PER) assay was performed basically as described in (Peng et al., 2015b). Two- to three-day-old fruit flies were starved overnight on wet Kim Wipe, briefly anaesthetized on ice, and affixed to a tooth pick. Fruit flies recovered in a humidified chamber for at least $1 \mathrm{~h}$ at room temperature before testing. Each subject was checked for intact PER before beginning the experiments. Flies that did not show the reflex were discarded. During the PER assay, the fruit fly was first satiated with water, and $100 \mathrm{~mm}$ sucrose with $10 \%$ DMSO containing 0 or $0.6 \mathrm{~mm}$ decanoic acid (WAKO) to activate SiHsTRPA was touched to the forelegs with a pipette tip. If the proboscis was extended and contact with the sucrose solution was maintained for $3 \mathrm{~s}$, the response was scored as 1 . If the contact of proboscis was brief, a 0.5 was awarded. If the proboscis failed to contact the solution within $5 \mathrm{~s}$ of offering, a 0 was awarded. Each fruit fly was offered compounds seven times per experiment, and between offerings water was given to satiation. Because decanoic acid was accepted on first offering (perhaps because the first activation of aversive taste neurons by SiHsTRPA was not sufficient to repress proboscis extension induced by activation of sugar taste neurons with 100 $\mathrm{mm}$ sucrose), PER frequency was calculated for the second through to the seventh offerings (sum of 6 scores per fruit fly divided by 6). Three groups of 7-8 flies per genotype were tested. For PER induction by decanoic acid, the forelegs of fruit flies prepared as above were touched with the $0.6-\mathrm{mm}$ solution in $10 \% \mathrm{DMSO}$, and then the extension of proboscis was scored followed by checking the intact PER by sucrose.

\section{GCaMP6 imaging in D. melanogaster}

For the GCaMP6 imaging, we first cut the female foreleg between the femur and the tibia and dipped the first three tarsal segments and the tibia in silicone oil. We then placed the severed legs laterally on double-sided tape 
pasted to a glass slide. Only the fourth and fifth tarsal segments were exposed and put under $5 \mu \mathrm{L}$ of $10 \%$ DMSO by covering the tibia and the first three tarsal segments of the leg with $2 \%$ agarose (Miyamoto et al., 2013). Three to four images were obtained first (0 s), and then time-lapse recording (every $0.5 \mathrm{~s}$ for $30 \mathrm{~s}$ ) by a CCD camera (RETIGA 2000-RV, Roper Scientific Photometrics) was started by adding $5 \mu \mathrm{L}$ of $1.2 \mathrm{~mm}$ decanoic acid in $10 \%$ DMSO. Data were acquired and analyzed by ImagePro Plus software (Media Cybernetics).

\section{Repellent assay with S. invicta in the laboratory}

Two filter papers were soaked with either 10\% DMSO or repellent in 10\% DMSO, and then briefly dried up on a paper towel. We joined two filter papers side by side and introduced $20 \mathrm{~S}$. invicta workers (briefly anaesthetized on ice) at the boundary at the beginning of the assay $(0 \mathrm{~min})$, and they were allowed to move for $40 \mathrm{~min}$ in the testing arena $(9-\mathrm{cm}$-diameter circle). The number of workers in the semicircle area with either DMSO or repellent was counted every $5 \mathrm{~min}$ for 10-40 min, and the avoidance index was calculated by the sum of seven recordings. Two filter papers soaked with $10 \%$ DMSO were used as a control assay. The experiments were repeated three times for each concentration of $\beta$-caryophyllene, $\beta$-citronellal, $\beta$-citronellol, octanoic acid, and decanoic acid shown in Fig. 8.

\section{Statistical analysis}

The results of Fig. 2B,C were analyzed by a two-tailed Dunnett test and two-tailed Steel test, respectively. The results of Fig. $8 B-E$ were analyzed by two-tailed Dunnett test. The results of Fig. 9A,C,D were analyzed by twotailed $t$ test. The results of calcium imaging in Figs. 2-7 were analyzed by one-tailed Welch's $t$ test.

\section{Results}

\section{Identification of SiHsTRPA and its mRNA expression} profile in S. invicta

Using AmHsTRPA sequence as a query, we searched for an ortholog in the annotated S. invicta gene set and genomic scaffolds and found two potential sequences. They represent S. invicta HsTRPA (SiHsTRPA) and Wtrw, respectively. Similar to other Hymenopteran insects, these two genes are located in the same genomic scaffold (04103) in tandem and are $3.7 \mathrm{~kb}$ apart. We obtained the full-length SiHsTRPA cDNA by using primers designed from the sequences of both $5^{\prime}$ and $3^{\prime}$ RACE products (accession no. LC188017). We did not identify any isoforms and obtained a single cDNA. SiHsTRPA contains seven ARs at the $\mathrm{N}$ terminus followed by the ion-transport domain with six transmembrane segments. This structure is common between HsTRPA channels including $\mathrm{AmH}-$ sTRPA (Kohno et al., 2010).

We examined the expression of SiHsTRPA mRNA in the abdomen, antennae, head, legs, and thorax of $S$. invicta by using reverse-transcription PCR (RT-PCR). 18S rRNA was equally present in all samples; however, SiHsTRPA mRNA was abundant in the antennae and legs compared with other tissues (Fig. 1A). These results were also confirmed by quantitative RT-PCR (qRT-PCR; Fig. 1B).

\section{SiHsTRPA protein expression in HEK293 cells}

Before characterizing the channel properties of SiHsTRPA, we first characterized the protein expression and cellular localization of V5-epitope-tagged SiHsTRPA in HEK293 cells. Specifically, we detected the protein with the expected molecular weight, $104 \mathrm{kDa}$, by Western blot analysis (Fig. 1C). We then examined the cellular localization of the epitope-tagged SiHsTRPA by double staining of the expressing cells with FITC-WGA (to label the plasma membrane) and anti-V5-epitope antibody. As shown in Fig. 1D, most of the protein was present slightly beneath the plasma membrane labeled by FITC-WGA; however, a fraction of the protein was localized at the plasma membrane.

\section{Heat-evoked activation of SiHsTRPA}

We measured the relative changes in intracellular calcium levels in HEK293 cells expressing SiHsTRPA by temperature fluctuations using a Fura-2 calcium imaging technique. Activation of the channel results in increasing the intracellular calcium levels by an influx of extracellular calcium. As shown in Fig. $2 A$, elevation but not reduction in temperature increased the relative intracellular calcium levels of cells expressing SiHsTRPA. Decanoic acid (C10) was used as a positive control to verify that SiHsTRPA expressed in the cell was functional. These results suggest that SiHsTRPA is a heat-activated TRPA channel.

\section{SiHsTRPA rescues the impaired thermotactic and thermal nociception behaviors of $D$. melanogaster trpA1 mutant}

The above results revealed that SiHsTRPA is activated by heat. We, therefore, tested the thermotactic behaviors of wild-type, $\operatorname{trp} A 1^{1}$ (Kwon et al., 2008), and $\operatorname{trp} A 1^{1}$ adults expressing DmTRPA1 or SiHsTRPA under trpA1-Gal4 (Rosenzweig et al., 2005; Kim et al., 2010) by using an aluminum block with a temperature gradient of $14-37^{\circ} \mathrm{C}$ (Sayeed and Benzer, 1996). This thermotactic behavior of adult $D$. melanogaster would depend on not only DmTRPA1 but also other thermosensors such as Pyx and Gr28b (Lee et al., 2005; Ni et al., 2013). Although wild-type adult fruit flies preferred temperatures $\sim 25-26^{\circ} \mathrm{C}$ and avoided temperatures $>28^{\circ} \mathrm{C}$ (Fig. $2 B$ ), $\operatorname{trp} A 1^{1}$ adults preferred high temperatures, and more flies were found in the test zones where the temperatures were $>28^{\circ} \mathrm{C}$ (Fig. $2 B$; Hamada et al., 2008). However, this behavioral defect was rescued by expressing either DITRPA1 or SiHsTRPA (Fig. 2B).

We also tested the thermal nociception behaviors of the wild-type, $\operatorname{trp} A 1^{1}$, and $\operatorname{trp} A 1^{1}$ larvae expressing either DITRPA1 or SiHsTRPA in the class IV multidendritic (mdlV) neurons under ppk1.9-Gal4 (Ainsley et al., 2008). For this, we characterized the stereotyped nocifensive escape locomotion behavior on touch, with a $46^{\circ} \mathrm{C}$ probe (Tracey et al., 2003; Neely et al., 2011; Zhong et al., 2012). The mdIV neurons function as the polymodal nociceptors of larvae. Most of the wild-type larvae initiated escape behavior within $1 \mathrm{~s}$ as previously reported; however, $\operatorname{trp} A 1^{1}$ larvae displayed a significantly delayed response 
A
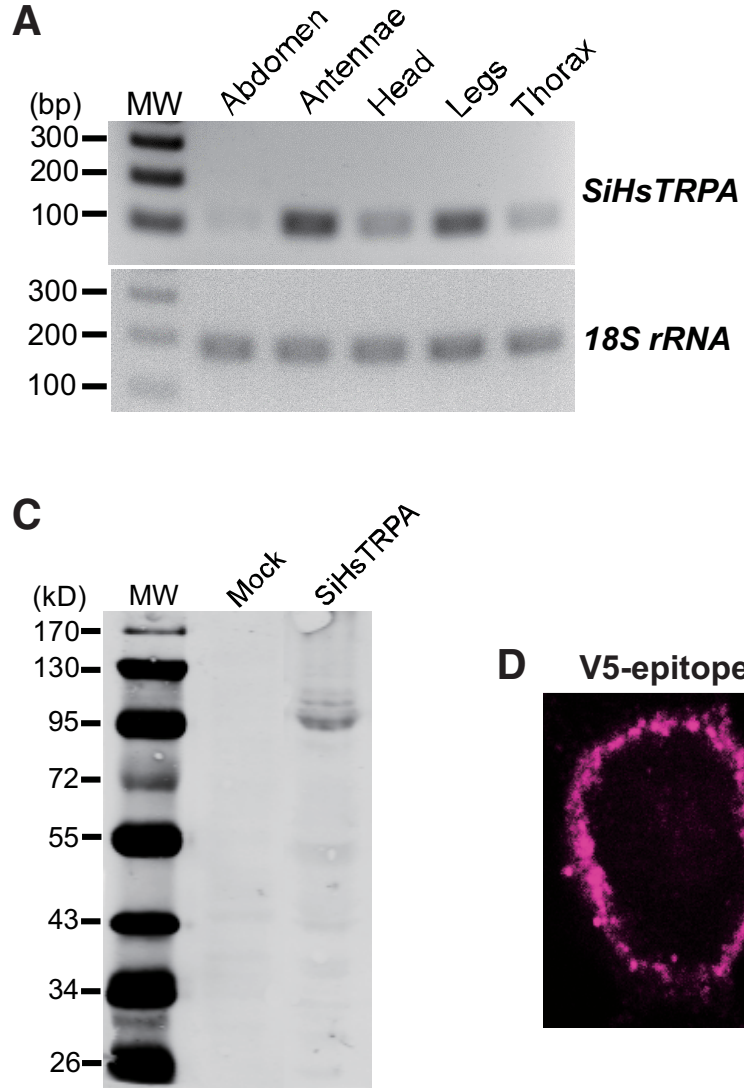

D
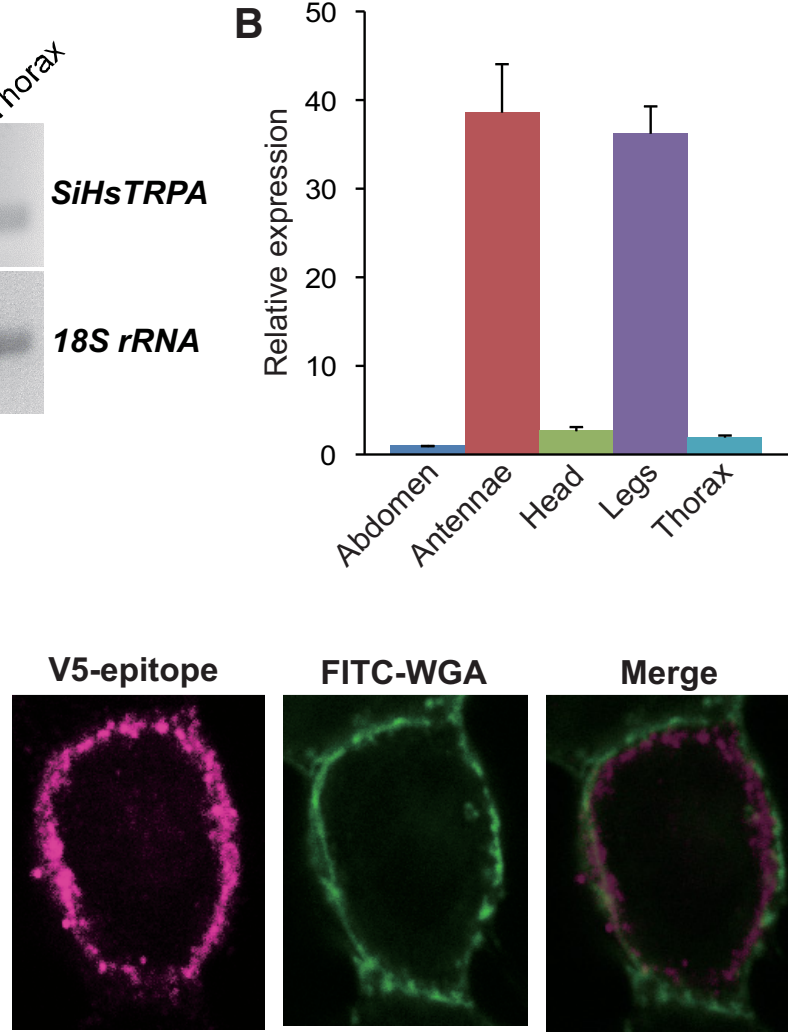

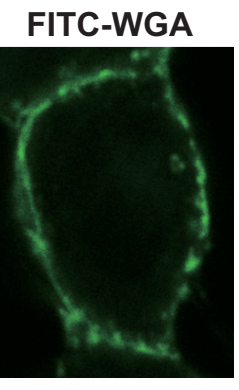

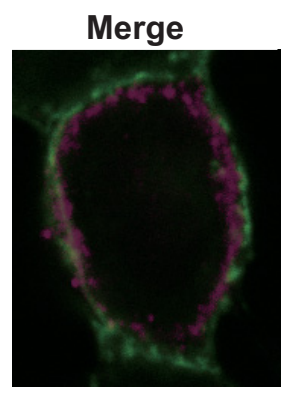

Figure 1. Expression profile of SiHsTRPA mRNA and the protein expression in HEK293 cells. A, Detection of SiHsTRPA mRNA and S. invicta 18S rRNA in the abdomen, antennae, head, legs, and thorax by RT-PCR. The position of 100-300-bp DNA molecular weight marker (MW) is shown at the left. $\boldsymbol{B}$, Relative expression levels of SiHsTRPA mRNA in the abdomen, antennae, head, legs, and thorax measured by qRT-PCR. S. invicta 18S rRNA was used as the internal standard, and the level in the abdomen was set as 1. C, Proteins expressed in HEK293 cells transfected with empty vector (Mock) and SiHsTRPA-expressing construct were analyzed by Western blot. The size (in kilodaltons) of protein molecular weight marker (MW) is at the left. $\boldsymbol{D}$, Localizations of plasma membrane-bound FITC-WGA and SiHsTRPA tagged with V5-epitope in the transfected HEK293 cells by immunofluorescence. The merged image is also shown.

(Fig. 2C; Neely et al., 2011; Zhong et al., 2012). In fact, many of the mutant larvae never showed the escape behavior (Fig. 2C). Introducing either DITRPA1 or SiHsTRPA improved the impaired nociceptive behavior of $\operatorname{trp} A 1^{1}$ larvae; however, SiHsTRPA appeared to rescue the phenotype to a lesser extent than did DmTRPA1. In fact, $>30 \%$ of the larvae showed response latency of $>10 \mathrm{~s}$ (Fig. 2C). Nevertheless, the $\operatorname{trp} A 1^{1}$ larvae expressing SiHsTRPA group did not show a statistically significant difference from the wild type. These results suggest that SiHsTRPA could complement the functions of DmTRPA1 together with Pain and Pyx (Tracey et al., 2003; Neely et al., 2011; Zhong et al., 2012) for thermal nociceptive behavior in fruit fly larvae.

\section{SiHsTRPA and AmHsTRPA show different chemical responses}

AmHsTRPA was shown to be activated by electrophilic compounds (allyl isothiocyanate and cinnamaldehyde) and camphor. However, no other AmHsTRPA-activating compounds were reported (Kohno et al., 2010). Because many plant-derived tick repellents were shown to activate the honey bee ectoparasitic mite TRPA1 channels (Peng et al., 2015b; Dong et al., 2016), we tested the effects of these repellents on activation of both SiHsTRPA and AmHsTRPA channels, using a calcium-imaging technique. We found that 19 compounds activated AmHsTRPA while only three compounds activated SiHsTRPA. The list of active and inactive compounds that open SiHsTRPA and AmHsTRPA channels is shown in Table 1. Fig. 3 shows the activation of SiHsTRPA and AmHsTRPA by cinnamaldehyde, $\beta$-citronellal, and $\beta$-caryophyllene. Cinnamaldehyde is an electrophilic compound (Macpherson et al., 2007), and $\beta$-citronellal has been used as insect repellent (Sakulku et al., 2009). $\beta$-Caryophyllene was shown to repel S. invicta (Wheeler et al., 2003; Kafle and Shih, 2013). We also identified two saturated fatty acids, decanoic acid (C10) and lauric acid (C12), as SiHsTRPAactivating compounds during an initial screen. We then tested the effects of saturated fatty acids with different numbers of carbon on SiHsTRPA (Figs. 4 and extended data Fig. 4-1). We found that saturated fatty acids with C3-C7 (propionic acid, butyric acid, valeric acid, hexanoic acid, and heptanoic acid) were inactive, but those with $>$ C8 (octanoic acid) were able to activate SiHsTRPA. These results demonstrate that a minimum of eight carbons are necessary for a saturated fatty acid to activate SiHsTRPA. Contrary to other compounds, the activation 
A
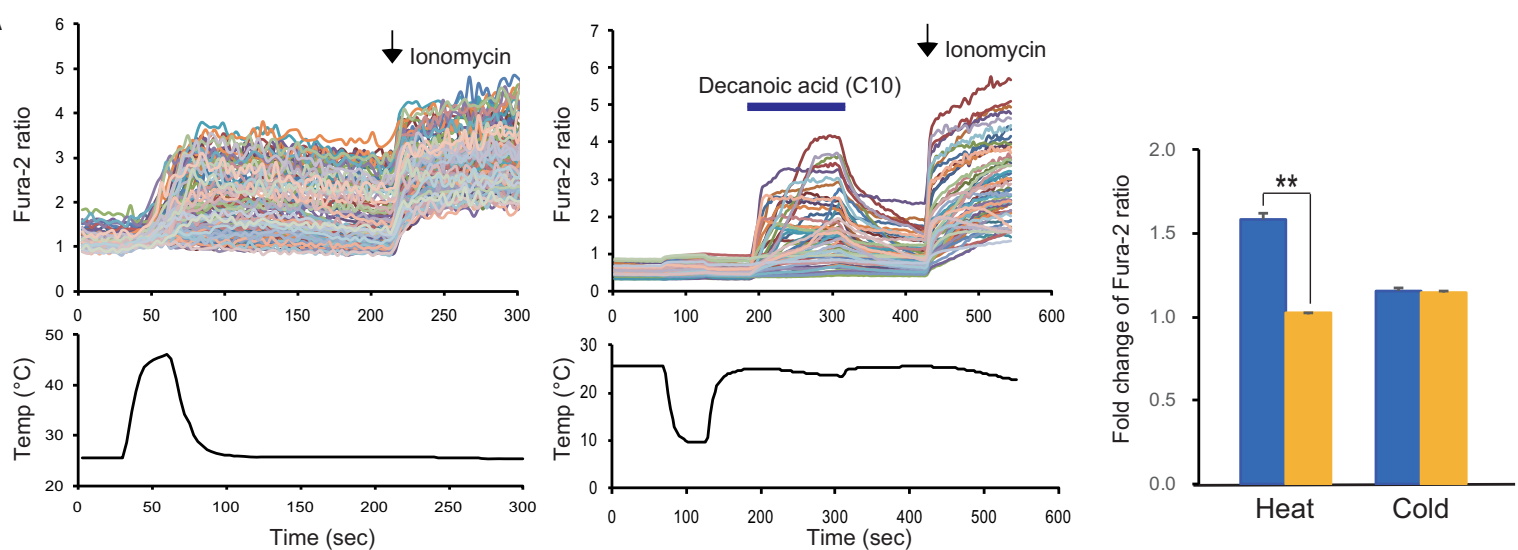

B

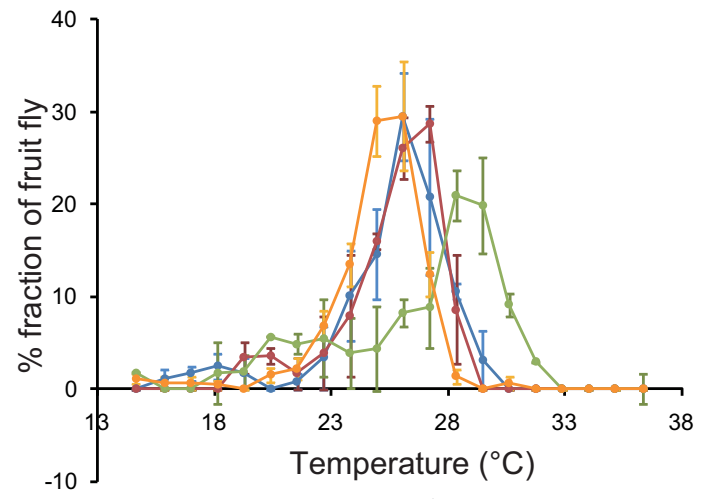

Wild type

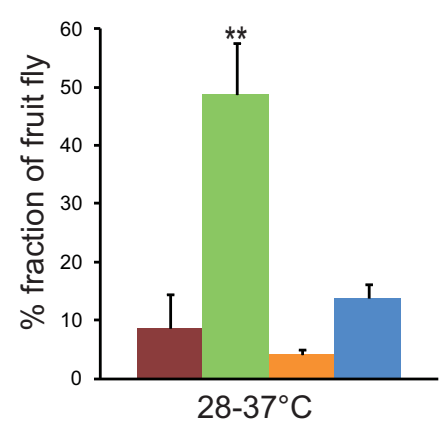

$\operatorname{trpA} 1>\operatorname{SiHsTRPA} ; \operatorname{trp} A^{1}$
C
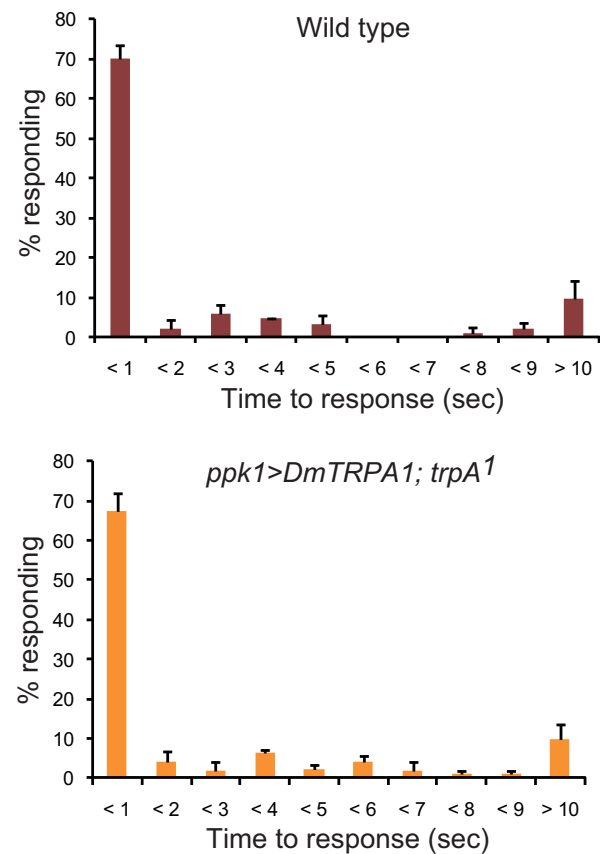
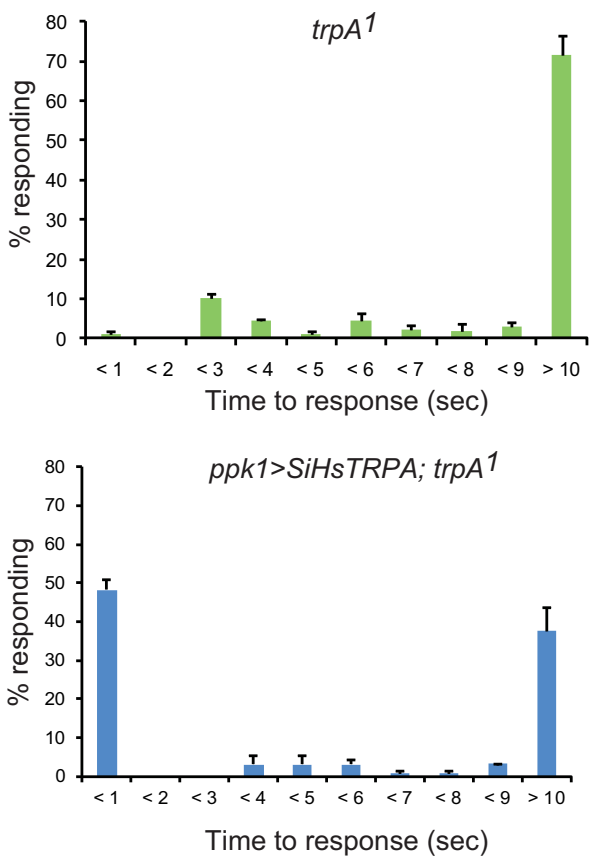

Figure 2. Heat activation of SiHsTRPA and the effects of introduction on the temperature-related phenotypes of $D$. melanogaster trp $A^{1}$ mutant. $\boldsymbol{A}$, The upper traces indicate the changes of intracellular calcium level in SiHsTRPA-expressing cells by high (left) and low (right) temperatures. The lower traces show the changes of bath temperature by time (sec, second). Each line represents the intracellular calcium level in the individual cell measured by calcium imaging. Decanoic acid (C10) was added at 180-300 s (blue bar) after cold stimulation to 
continued

confirm that the channels expressed in the cells were functional. The arrows show the time points when $5 \mu \mathrm{m}$ ionomycin was added. The mean value of fold change of Fura-2 ratio with error bar ( \pm SEM) is shown for the heat- or cold stimulated DsRed-positive (blue) and DsRed-negative (yellow) cells. The total numbers of cells analyzed were 79-228. Significant difference is shown by (**, $P<0.001$, one-tailed Welch's $t$ test). $B$, The distribution of wild-type, trpA $1^{1}$, and trpA $1^{1}$ expressing either DmTRPA1 (trpA1 > DmTRPA1; trpA ${ }^{1}$ ) or SiHsTRPA (trpA1 > SiHsTRPA; $\left.\operatorname{trp} A 1^{1}\right)$ under trpA1-Gal4 was recorded along a thermal gradient $\left(14-37^{\circ} \mathrm{C}\right)$. The recording was repeated three times for each group. The mean value with error bar ( $\pm \mathrm{SEM} ; n=3$ ) is shown for each temperature section (left). The percentage of fruit flies in the area of $28-37^{\circ} \mathrm{C}$ (wild type: 8.6 \pm 5.9\%; $\operatorname{trp}^{1} 1^{1}: 48.8 \pm 8.9 \%$; $\operatorname{trp}^{1} 1^{1}$ expressing DITRPA1: $4.1 \pm 0.9 \%$; trpA $1^{1}$ expressing SiHsTRPA: $13.7 \pm 2.6 \%$ ) of the thermal gradient. Only $\operatorname{trp} A 1^{1}(* *)$ is significantly different from wild type (two-tailed Dunnett test, $P<0.003$; right). C, Wild type $(n=92)$, trpA $1^{1}$ ( $\left.n=90\right)$, and $\operatorname{trp} A 1^{1}$ expressing either DmTRPA1 (ppk1 > DmTRPA1; trpA1 ${ }^{1}, n=83$ ) or SiHsTRPA (ppk1 > SiHsTRPA; trpA1 ${ }^{1}, n=125$ ) under ppk1.9-Gal4 were tested for their response to high temperature using a $46^{\circ} \mathrm{C}$ probe. The percentage of responding larvae for each genotype is shown at each second within $9 \mathrm{~s}$ and $>10 \mathrm{~s}$ (mean value \pm SEM). P-values (two-tailed Steel test) for wild type and trpA $1^{1}$ expressing either DmTRPA1 or SiHsTRPA compared to trpA $1^{1}$ are $<0.023,<0.023$, and $<0.038$, respectively. $P$-value (two-tailed Steel test) for trpA $1^{1}$ expressing $\operatorname{SiHsTRPA}$ is $<0.12$ compared with wild type.

by fatty acids is quite similar between AmHsTRPA and SiHsTRPA (Fig. 4 and extended data Fig. 4-2).

As shown in Fig. 5, $\beta$-citronellol, camphor, diallyl disulfide, nerol, and thujone activated AmHsTRPA but not SiHsTRPA (extended data Fig. 5-1). In fact, most AmHsTRPA-activating compounds did not activate $\mathrm{SiH}-$ STRPA (Table 1). Thus, chemical activation profiles are quite different between these two HsTRPA orthologs of honey bee and RIFA, which belong to the same insect order, Hymenoptera. Intriguingly, $\beta$-citronellal with a car- bonyl group activated both SiHsTRPA and AmHsTRPA (Fig. 3); however, $\beta$-citronellol with a hydroxyl group activated only AmHsTRPA (Fig. 5).

\section{Amino acids in the multiple domains of AmHsTRPA are necessary for the response to diallyl disulfide and camphor}

Alignment of HsTRPA amino acid sequences from jewel wasp, leaf cutting bee, honey bee, bumble bee, and seven ants revealed that the $\mathrm{N}$-terminal sequences of bee

Table 1. List of active and inactive compounds to open SiHsTRPA and AmHsTRPA channels

\begin{tabular}{|c|c|c|c|}
\hline $\begin{array}{l}\text { Active compounds } \\
\text { to open SiHsTRPA }\end{array}$ & $\begin{array}{l}\text { Inactive compounds } \\
\text { to open SiHsTRPA }\end{array}$ & $\begin{array}{l}\text { Active compounds } \\
\text { to open AmHsTRPA }\end{array}$ & $\begin{array}{l}\text { Inactive compounds } \\
\text { to open AmHsTRPA }\end{array}$ \\
\hline $\begin{array}{l}\text { Fatty acids (C8, 1.32; C9, } \\
\text { 1.619; C10, 1.596; C11, 1.93; } \\
\text { C12, 1.991) }\end{array}$ & Fatty acids, C3-C7 & $\begin{array}{l}\text { Fatty acids (C8, 1.108; } \\
\quad \text { C9, 1.89; C10, 1.973; C11, 2.15; } \\
\text { C12, 2.464) }\end{array}$ & $3 \mathrm{~mm}$ menthol \\
\hline Cinnamaldehyde (1.146) & Diallyl disulfide & Cinnamaldehyde (1.678) & $0.5 \mathrm{~mm}$ carvacrol \\
\hline$\beta$-Citronellal (1.704) & $\beta$-Citronellol & Diallyl disulfide (1.216) & $\alpha$-Terpineol \\
\hline \multirow[t]{23}{*}{$\beta$-Caryophelene (1.342) } & Camphor & $\beta$-Citronellal (2.681) & 2-Undecanone \\
\hline & $0.5 \mathrm{~mm}$ geranylacetone & $\beta$-Citronellol (1.634) & Terpinen-4-ol \\
\hline & Eugenol & $\beta$-Caryophelene (3.228) & $0.1 \%$ creosote \\
\hline & Nerolidol & Camphor (1.421) & o-Methoxyphenol \\
\hline & $0.5 \mathrm{~mm}$ nerol & $0.5 \mathrm{~mm}$ nerol (1.311) & 2-Methoxy-4-methylphenol \\
\hline & Thujone & Thujone (1.432) & $\begin{array}{l}N, N \text {-diethyl-2- } \\
\text { phenylacetamide }\end{array}$ \\
\hline & Myrtenal & Nerolidol & Methyl salicylate \\
\hline & $\beta$-Cyclocitral & $0.5 \mathrm{~mm}$ geranylacetone & Verbenone \\
\hline & 2-Dodecanone & Eugenol & Coumarin \\
\hline & 2-Ethyl-1,3-hexanediol & Myrtenal & Thymol \\
\hline & Methyl jasmonate & $\beta$-Cyclocitral & \\
\hline & $3 \mathrm{~mm}$ menthol & 2-Dodecanone & \\
\hline & $0.5 \mathrm{~mm}$ carvacrol & 2-Ethyl-1,3-hexanediol & \\
\hline & $\alpha$-Terpineol & Methyl jasmonate & \\
\hline & 2-Undecanone & Geraniol & \\
\hline & Terpinen-4-ol & Borneol & \\
\hline & Thymol & AITC & \\
\hline & 2-Methoxy-4-methylphenol & & \\
\hline & Geraniol & & \\
\hline & o-Methoxyphenol & & \\
\hline & Coumarin & & \\
\hline & Verbenone & & \\
\hline & $\begin{array}{l}0.1 \% \text { Creosote; methyl salicylate; } \\
\text { borneol; } N, N \text {-diethyl-2- }\end{array}$ & & \\
\hline
\end{tabular}

All compounds were tested at 1-mm concentration except carvacrol, creosote, geranylacetone, nerol, and menthol, at the indicated concentrations. The average fold changes of Fura-2 ratio induced by active compounds shown in this report are indicated with parentheses. 

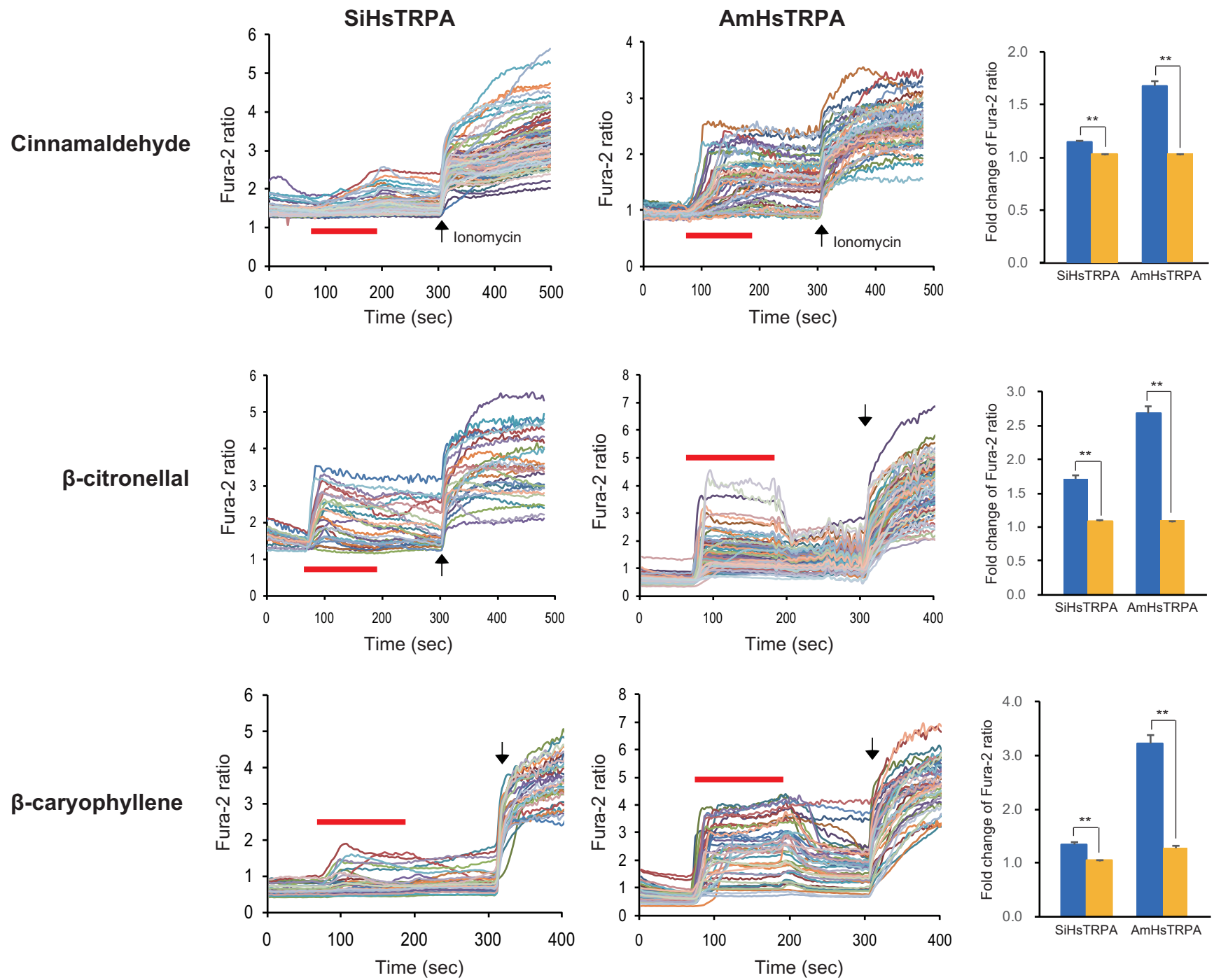

Figure 3. Activation of both SiHsTRPA and AmHsTRPA by three plant-derived compounds. Activation of both SiHsTRPA and AmHsTRPA by cinnamaldehyde, $\beta$-citronellal, and $\beta$-caryophyllene was analyzed by calcium imaging. Red bars show the period when each compound was added, and then washed off. Arrows show the time points when ionomycin was added. The concentration of each compound tested was $1 \mathrm{~mm}$. The mean value of fold change of Fura-2 ratio with error bar ( \pm SEM) is shown for the compound-stimulated DsRed-positive (blue) and DsRed-negative (yellow) cells expressing SiHsTRPA or AmHsTRPA. The total numbers of cells analyzed were 70-206. **, Significant difference by one-tailed Welch's $t$ test $(P<0.001)$.

HsTRPA channels are longer than those of the HsTRPA channels of the jewel wasp and ants (extended data Fig. $6-1)$. It was previously reported that the long $\mathrm{N}$-terminal sequence of the honey bee mite, Tropilaelaps mercedesae, TRPA1 (TmTRPA1) isoform plays a critical role in chemical sensitivity. The TmTRPA1 isoform with a short $\mathrm{N}$-terminal sequence responded to fewer compounds (Dong et al., 2016). The same observation was also made with D. melanogaster TRPA1 (Kang et al., 2012; Du et al., 2015). We therefore tested the effects of deleting the $\mathrm{N}$-terminal sequence of AmHsTRPA (amino acids 21-98) and adding the sequence (amino acid 1-98) to SiHsTRPA. The N-terminal deletion mutant of AmHsTRPA (AmHsTRPA $\Delta N$ ) was weakly activated by decanoic acid, but not by $5 \mathrm{~mm}$ camphor. Meanwhile, decanoic acid, but not camphor, activated SiHsTRPA with the $\mathrm{N}$-terminal sequence of AmHsTRPA added (AmHsTRPA-N + SiHsTRPA; Fig. 6).
These results demonstrate that the $\mathrm{N}$-terminal sequence is necessary but not sufficient to support the full chemical activation of AmHsTRPA. In fact, we found that both camphor and diallyl disulfide robustly activate NvHsTRPA, which lacks the extended $\mathrm{N}$-terminal sequence and outgroups both AmHsTRPA and SiHsTRPA (Fig. 6 and extended data Figs. 6-1 and 6-2). These results suggest that amino acid substitutions in SiHsTRPA have resulted in the loss of reactivity to the above compounds.

To identify the protein domains of AmHsTRPA critical for the responses to diallyl disulfide and camphor, we constructed nine AmHsTRPA chimeric channels in which the specific domains with relatively low sequence similarity were swapped between AmHsTRPA and SiHsTRPA (Fig. 7A). All chimeric proteins were equally expressed in HEK293 cells (extended data Fig. 7-3), and their responses to the above compounds, as well as decanoic 

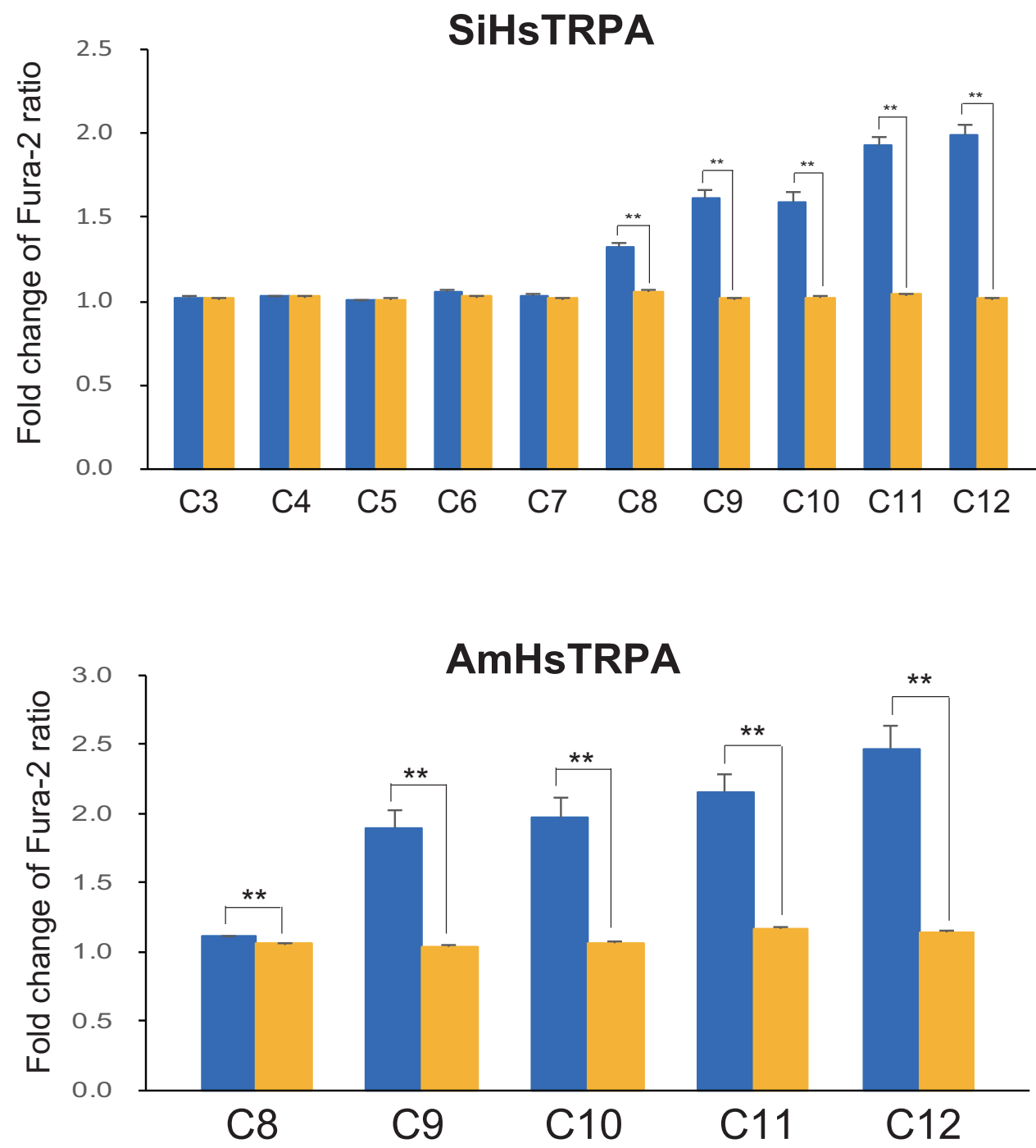

Figure 4. Activation of SiHsTRPA and AmHsTRPA by saturated medium-chain fatty acids. Activation of SiHsTRPA and AmHsTRPA by saturated fatty acids was analyzed by calcium imaging. The mean value of fold change of Fura-2 ratio with error bar ( \pm SEM) is shown for the fatty acid (propionic acid, C3; butyric acid, C4; valeric acid, C5; heptanoic acid, C6; hexanoic acid, C7; octanoic acid, C8; nonanoic acid, C9; decanoic acid, C10; undecanoic acid, C11; and lauric acid, C12) stimulated DsRed-positive (blue) and DsRed-negative (yellow) cells. The total numbers of SiHsTRPA- and AmHsTRPA-transfected cells analyzed were 97-212 and 61-157, respectively. **, Significant difference by one-tailed Welch's $t$ test $(P<0.001)$. The traces in extended data Figs. 4-1 and 4-2 indicate the changes of intracellular calcium level in SiHsTRPA- and AmHsTRPA-expressing cells by above fatty acids, respectively.

acid, were tested (Fig. $7 B$ and extended data Figs. 7-1 and 7-2). Because the reactivity of selectivity filter and $C$ terminus-swapped chimeras to decanoic acid was obscure after stimulation by either diallyl disulfide or camphor (extended data Fig. 7-2), we tested the responses of these two chimeras to decanoic acid alone (extended data Fig. 7-2, bottom panels). Amino acids in the AR2 of AmHsTRPA were necessary for the responses to camphor, diallyl disulfide, and decanoic acid, and the responses of chimeric channels to camphor and decanoic acid were similar (Fig. 7B and extended data Figs. 7-1 and 7-2). Thus, the same protein domains of AmHsTRPA have important roles for responding to the above two compounds. In contrast, amino acids in ARs 3 and 5-7 of AmHsTRPA were specifically required for the response to diallyl disulfide (Fig. 7B and extended data Figs. 7-1 and
7-2). These results suggest that camphor and decanoic acid share the same activation mechanism for $\mathrm{AmH}$ STRPA, which is expectedly different from the mechanism of an electrophile, diallyl disulfide.

\section{SiHsTRPA-activating compounds repel $S$. invicta}

As shown in Fig. 3, SiHsTRPA was activated by cinnamaldehyde and insect repellents, suggesting that it functions as a sensor for noxious compounds. We thus tested whether SiHsTRPA-activating compounds act as repellents for S. invicta in the laboratory. We tested the behavior of S. invicta placed on two adjacent filter papers soaked with either $10 \%$ DMSO or a testing compound in $10 \%$ DMSO. In the control assay, both filter papers were soaked with $10 \%$ DMSO (Fig. $8 A$ ). SiHsTRPA-activating compounds, $\beta$-caryophyllene (Fig. $8 B$ ), octanoic acid (Fig. 

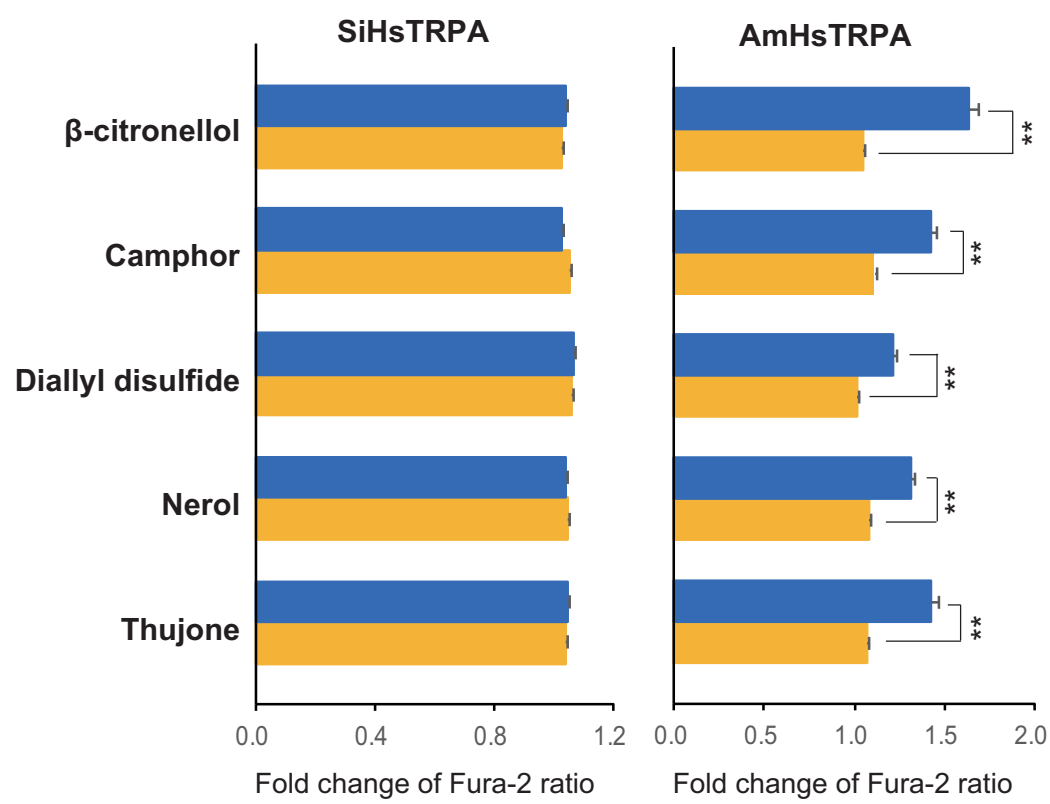

Figure 5. Five plant-derived compounds activate AmHsTRPA but not SiHsTRPA. Responses of SiHsTRPA and AmHsTRPA to $\beta$-citronellol, camphor, diallyl disulfide, nerol, and thujone were tested by calcium imaging. The mean value of fold change of Fura-2 ratio with error bar ( \pm SEM) is shown for the compound-stimulated DsRed-positive (blue) and DsRed-negative (yellow) cells. The total numbers of SiHsTRPA- and AmHsTRPA-transfected cells analyzed were 73-153 and 71-103, respectively. **, Significant difference by one-tailed Welch's $t$ test $(P<0.001)$. The traces in extended data Fig. 5-1 indicate the changes of intracellular calcium level in SiHsTRPA- and AmHsTRPA-expressing cells by above five compounds.

$8 C$ ), decanoic acid (Fig. 8D), and $\beta$-citronellal (Fig. 8E, purple) repelled $S$. invicta in a concentration-dependent manner. $\beta$-Citronellol, which did not activate SiHsTRPA despite its almost identical structure to $\beta$-citronellal (Fig. 5), was unable to repel $S$. invicta (Fig. $8 E$, blue). Thus, the repellent activity of a compound reflects its ability to activate SiHsTRPA. These results demonstrate that SiHsTRPAactivating compounds function as repellents against $S$. invicta, and SiHsTRPA is a sensor for noxious compounds.

\section{Activation of SiHsTRPA is sufficient to modify feeding behavior of $D$. melanogaster}

To test whether the activation of SiHsTRPA by decanoic acid is sufficient to repress feeding behavior, we characterized the gustatory responses of $D$. melanogaster expressing SiHsTRPA and a calcium-sensor protein, GCaMP6 (Chen et al., 2013) under Gr33a-Gal4, to a 100-mm sucrose solution containing $0.6 \mathrm{~mm}$ decanoic acid by PER (Kohno et al., 2010; Peng et al., 2015b). D. melanogaster Gr33a is a gustatory receptor gene widely expressed in aversive taste neurons (Moon et al., 2009). PER frequency was comparable between fruit flies expressing GCaMP6 alone and both $\mathrm{SiH}$ STRPA and GCaMP6 with $100 \mathrm{~mm}$ sucrose. However, it was significantly reduced in the presence of $0.6 \mathrm{~mm}$ decanoic acid for the animals expressing both SiHsTRPA and GCaMP6 (Fig. 9A). These results demonstrate that in $D$. melanogaster, direct chemical activation of SiHsTRPA in aversive taste neurons could suppress feeding behavior elicited by sucrose.

We also tested whether stimulation of SiHsTRPA in the sugar taste neurons by $0.6 \mathrm{~mm}$ decanoic acid was sufficient to induce feeding behavior. We expressed both SiHsTRPA and GCaMP6 in Gr64f-positive sugar taste neurons (Jiao et al., 2008), and GCaMP6 fluorescence was detected in several discrete neurons in the distal segments of the foreleg (Peng et al., 2015b). As shown in Fig. $9 B$, applying $0.6 \mathrm{~mm}$ decanoic acid to the distal segments of foreleg increased GCaMP6 fluorescence in the Gr64f-expressing sugar taste neurons associated with multiple sensilla, including 5D1, 5V1, and 5V2 sensilla (Miyamoto et al., 2013). We detected an increase in intracellular calcium level $\left(\Delta F / F_{0}\right)$ in the sugar taste neuron associated with 5D1 sensilla expressing both SiHsTRPA and GCaMP6, but not expressing GCaMP6 alone (Fig. 9C). SiHsTRPA stimulation by decanoic acid was able to activate the sugar taste neurons, and as a result, the application of $0.6 \mathrm{~mm}$ decanoic acid to the forelegs was sufficient to induce significant PER in the fruit flies expressing both SiHsTRPA and GCaMP6, but not GCaMP6 alone (Fig. 9D). These results demonstrate that manipulating the activity of taste neurons by the introduction of SiHsTRPA and by the application of decanoic acid is sufficient to modify the gustatory behaviors of fruit flies.

\section{Discussion}

\section{S. invicta highly expresses SiHsTRPA in the antennae and legs}

We first identified a Wtrw ortholog and its duplicated gene, SiHsTRPA, in an S. invicta genomic scaffold. Consistent with the lack of introns in the SiHsTRPA gene, we isolated the single cDNA that encodes a TRPA channel with seven ARs and an ion-transport domain containing six transmembrane segments. These structural features appear to be shared between HsTRPA channels of bees, wasps, and ants. Nevertheless, duplicated Wtrw genes 

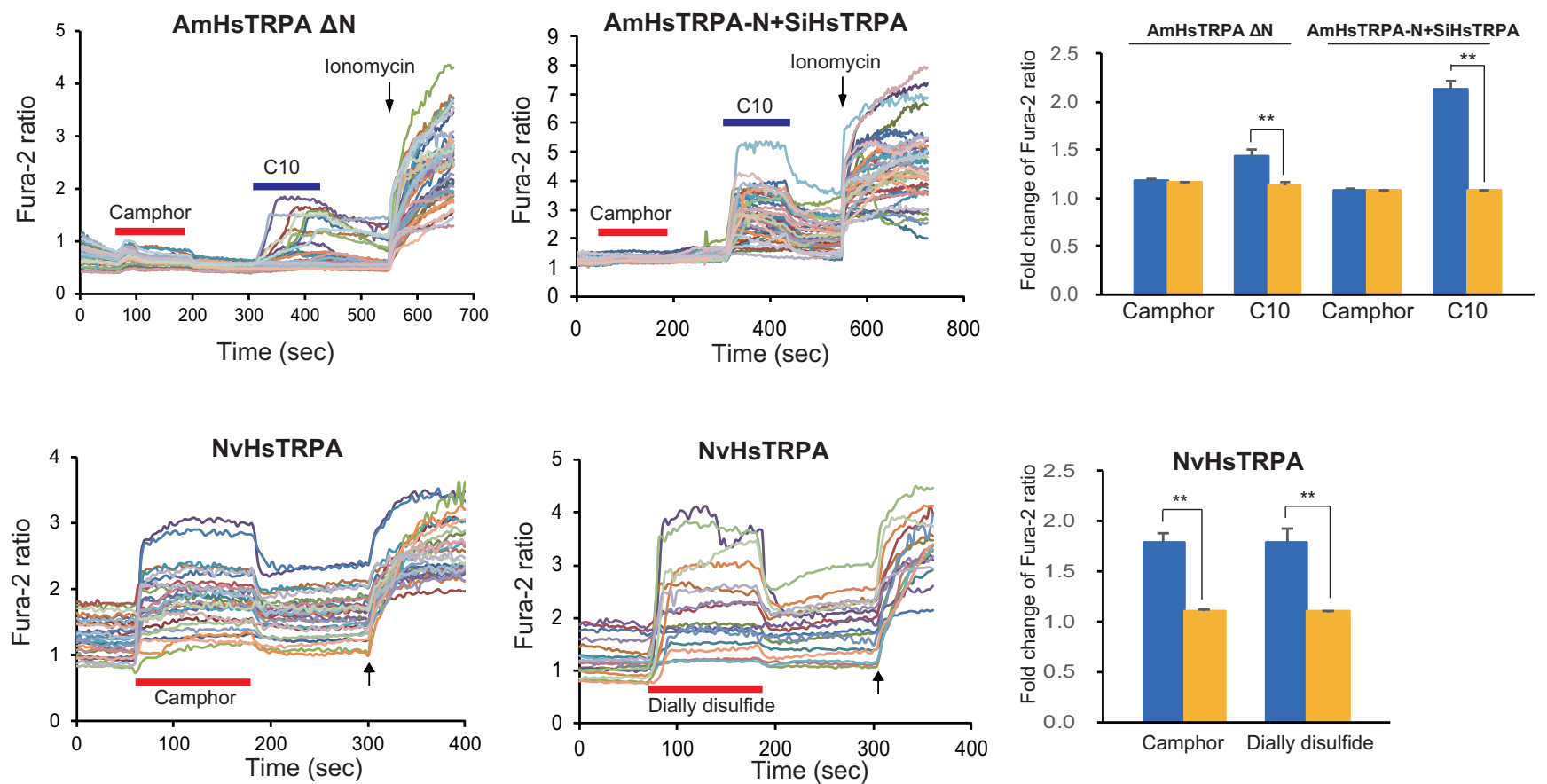

Figure 6. Camphor activates NvHsTRPA but not N-terminal-deleted AmHsTRPA mutant and SiHsTRPA added with $\mathrm{N}$ terminus of AmHsTRPA. Responses of N-terminal-deleted AmHsTRPA mutant (AmHsTRPA $\Delta N$ ), SiHsTRPA added with $\mathrm{N}$ terminus of AmHsTRPA (AmHsTRPA-N + SiHsTRPA), and NvHsTRPA to camphor and diallyl disulfide were tested by calcium imaging. Red bars show the period when each compound was added, and then washed off. C10 was applied to the mutants after testing camphor as shown by blue bars. Arrows show the time points when ionomycin was added. The mean value of fold change of Fura-2 ratio with error bar ( \pm SEM) is shown for the compound stimulated DsRed-positive (blue) and -negative (yellow) cells. The total numbers of AmHsTRPA $\triangle \mathrm{N}-$, AmHsTRPA-N + SiHsTRPA-, and NvHsTRPA-transfected cells analyzed were 100-114, 104-109, and 46-90, respectively. **, Significant difference by one-tailed Welch's $t$ test $(P<0.001)$. Amino acid sequence alignment of wasp, bee, and ant HsTRPA channels is shown in extended data Fig. 6-1. Molecular phylogenetic tree of wasp, bee, and ant HsTRPA channels is indicated in extended data Fig. 6-2.

are also present in Bombyx mori (silk moth), Manduca sexta (tobacco hornworm), and Acyrthosiphon pisum (pea aphid) genomes, suggesting that the duplication occurred in the common ancestor of insects but the duplicated gene has been lost in many insect species. The retention of HsTRPA in Hymenoptera would be essential because it has to replace the functions of the lost ancient TRPA1 (Matsuura et al., 2009; Kohno et al., 2010). SiHsTRPA mRNA is likely to be expressed in sensory neurons associated with various types of sensilla (Renthal et al., 2003; Ramirez-Esquivel et al., 2014). These results may suggest that SiHsTRPA functions as a peripheral sensor to detect stimuli in the environment based on our in vitro and heterologous expression experiments. Thus, SiHsTRPA appears to play a major role for sensory perception through sensilla in the antennae and legs.

\section{SiHsTRPA functions as a heat sensor}

Both SiHsTRPA and DmTRPA1 are heat sensitive and share similar heat-associated physiological functions. Nevertheless, heat-sensitive SiHsTRPA did not completely rescue the impaired thermal nociception behavior of trpA1 mutant larvae. This may suggest that DmTRPA1 plays other roles in addition to a thermal sensor for the thermal nociception behavior as previously reported (Zhong et al., 2012). Considering complex behaviors of ants, they should require thermosensors to avoid extreme tempera- tures, choose the preferred temperatures, and monitor the nest temperature for brood care. Some species may use temperature information as the cue for other behaviors (Ruchty et al., 2010b). Sensilla ampullaceal, sensilla coeloconica, and coelocapitular sensilla are considered to contain thermosensitive neurons (Ramirez-Esquivel et al., 2014); however, only cold-sensitive neurons have been identified in any ant to date (Ruchty et al., 2010a; Nagel and Kleineidam, 2015). Because several warm-sensitive glomeruli were identified in the antennal lobe of the leaf-cutting ant (Ruchty et al., 2010b), heat-sensitive neurons expressing SiHsTRPA are likely to be present in S. invicta.

\section{Evolutionary plasticity of the HsTRPA channel}

Interestingly, NvHsTRPA is activated by camphor and diallyl disulfide, which are incapable of activating SiHsTRPA. These results suggest that AmHsTRPA and NvHsTRPA share a common chemical sensitivity, and based on the molecular species tree of wasps, bees, and ants (Gadau et al., 2012), as well as the phylogenetic tree of HsTRPA channels, SiHsTRPA appears to have lost most of the chemical sensitivity of the common ancestor of HsTRPA. In fact, a number of repellents for $S$. invicta and other ants have been identified previously (Dani et al., 1996; Wheeler et al., 2003; Dutcher and Beaver, 2005; Chen et al., 2008; Kafle and Shih, 2013; Zhang et al., 2014; Fu et al., 2015; Huang et al., 2015), but we found that most of them did 
A

AR

TS

AmHsTRPA $1: 2: 3: 4: 5: 6: 7$ 1.2 । 3 , 4 , $5=6$ N-TER $1: 2: 3: 4: 5: 6: 7$

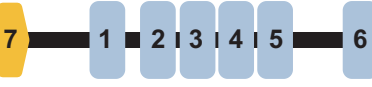
6

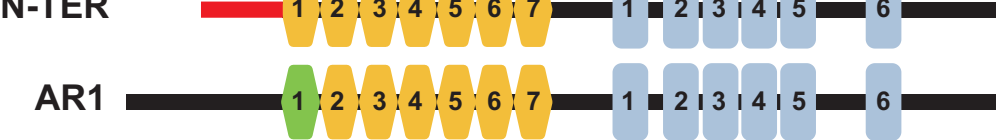

AR2 पी:

AR3

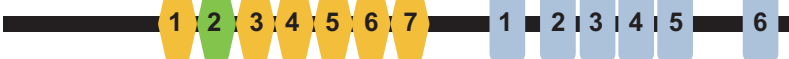

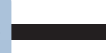

AR3

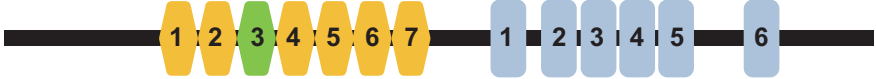

AR3-5

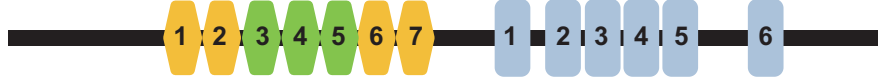

AR5-7

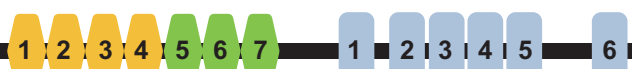

AR/TS
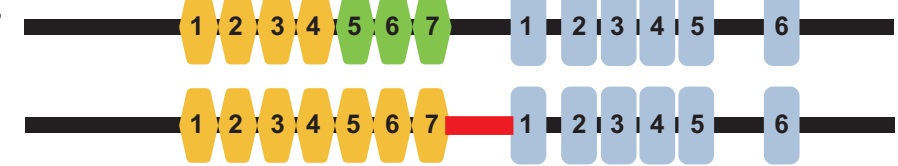
SF

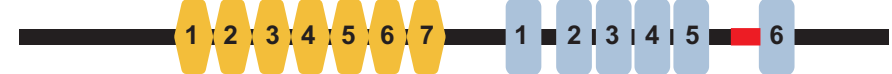

C-TER

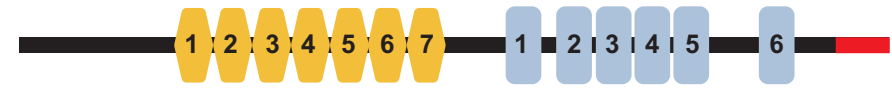

SiHsTRPA

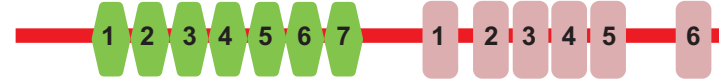

B

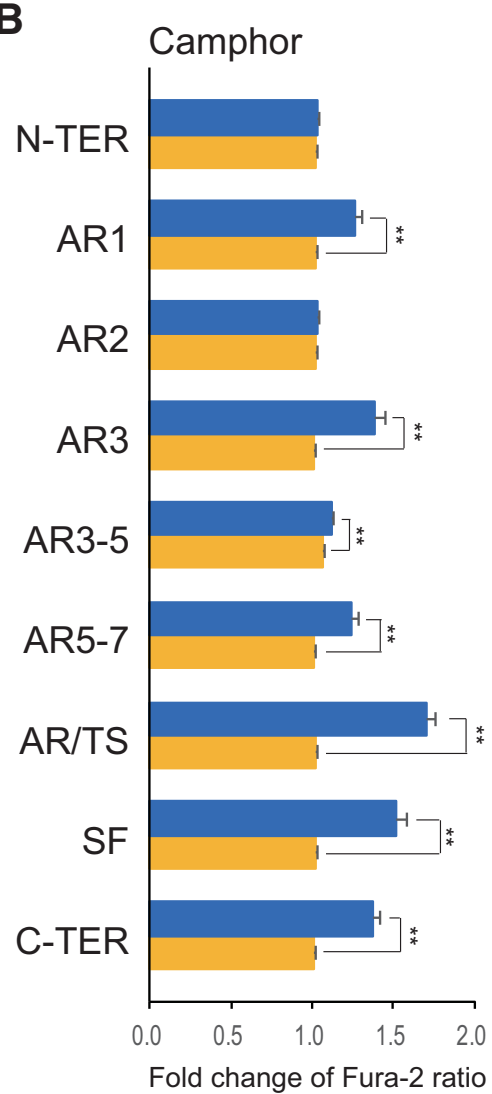

Decanoic acid

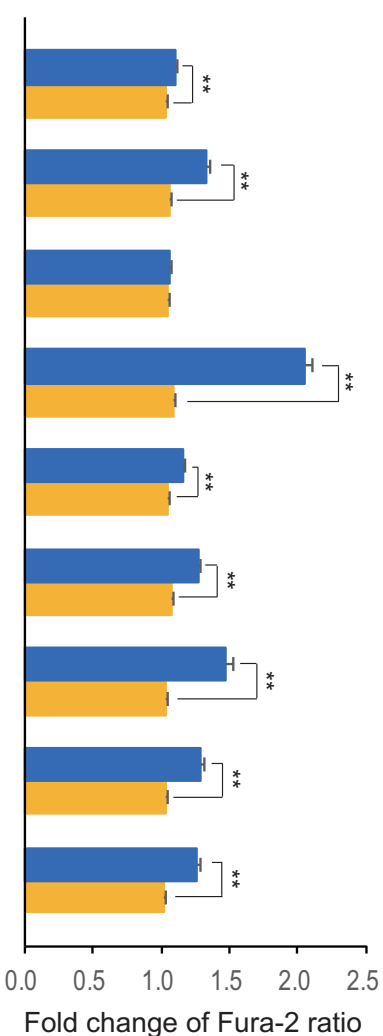

Diallyl disulfide

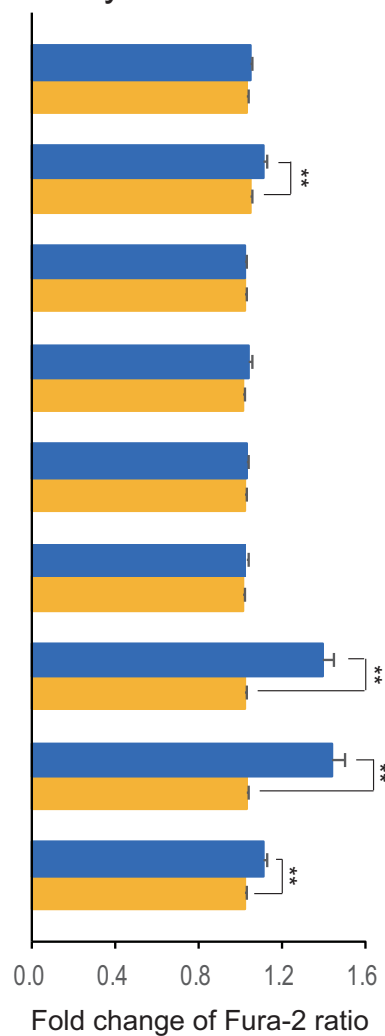


Figure 7. Responses of nine AmHsTRPA/SiHsTRPA chimeric channels to camphor, diallyl disulfide, and decanoic acid. A, Schematic representation of nine AmHsTRPA/SiHsTRPA chimeras as well as the parental AmHsTRPA and SiHsTRPA channels. Ankyrin repeat (AR) and transmembrane segment (TS) are shown by hexagon and rectangle, respectively. All chimeras were based on AmHsTRPA in which the $\mathrm{N}$ terminus (N-TER), AR1, AR2, AR3, AR3-5, AR5-7, the boundary between ARs and TS (AR/TS), the selectivity filter (SF), and the $\mathrm{C}$ terminus (C-TER) was swapped by the corresponding domain of SiHsTRPA. $\boldsymbol{B}$, The mean value of fold change of Fura-2 ratio with error bar ( \pm SEM) is shown for the compound-stimulated DsRed-positive (blue) and DsRed-negative (yellow) cells. The total numbers of camphor-, diallyl disulfide-, and decanoic acid-stimulated cells analyzed were 91-124, 87-122, and 180-246, respectively. **, Significant difference by one-tailed Welch's $t$ test $(P<0.001)$. The traces in extended data Fig. 7-1 and 7-2 indicate the changes of intracellular calcium level in cells expressing above AmHsTRPA/SiHsTRPA chimeric channel by camphor, diallyl disulfide, or C10. Protein expression of nine AmHsTRPA/SiHsTRPA chimeric channels is shown in extended data Fig. 7-3.

not activate SiHsTRPA (Table 1). Several examples are camphor, eugenol, cineole, nerolidol, butyl carbitol acetate, linalool, citral, and limonene. The loss of chemical reactivity of SiHsTRPA might be compensated by the increased number of Pain and/or TRPA5 genes in the S. invicta genome (Peng et al., 2015a) or other sensors/ receptors. Thus, this is another example that shows the evolutionary plasticity of TRP channels (Kadowaki, 2015; Saito and Tominaga, 2015).

The responses of AmHsTRPA/SiHsTRPA chimeric channels to diallyl disulfide, camphor, and decanoic acid provide insights into the mechanisms for activation of parent channels by these compounds. Although decanoic acid activated both AmHsTRPA and SiHsTRPA, it did not appear to fully activate the $\mathrm{N}$ terminus, AR2, and AR4 swapped chimeras. These results suggest that the amino acids in above protein domains have coevolved and thus closely interact with each other in the three-dimensional structure (Ovchinnikov et al., 2015). The protein structure formed by the above three domains, and perhaps other domains (for example, the transmembrane segments) would be responsible for interacting with decanoic acid and gating the channel. Alternatively, it would be involved in opening the gate after decanoic acid interacts with the other domain. Intriguingly, the same protein structure is also responsible for activation by camphor; however, this
A

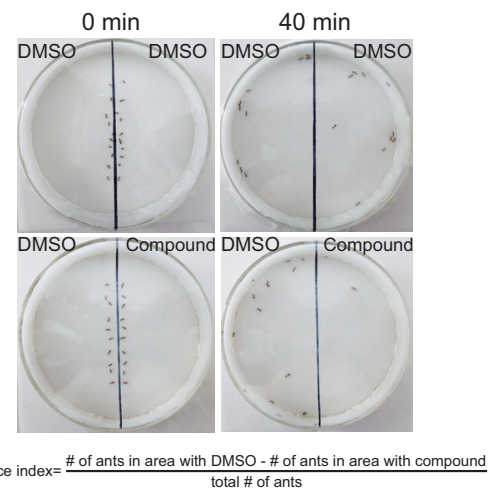

D

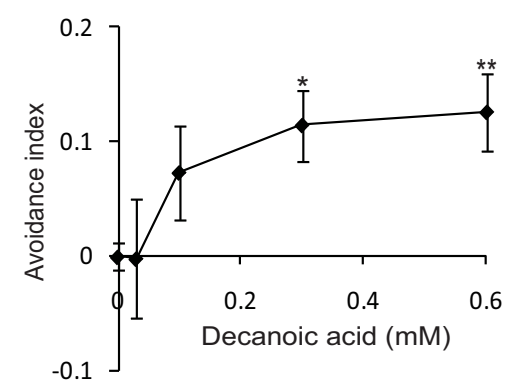

B

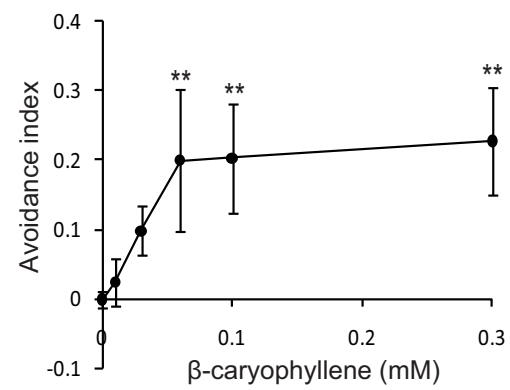

E

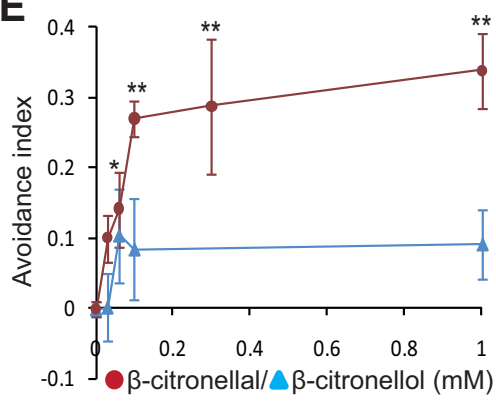

C

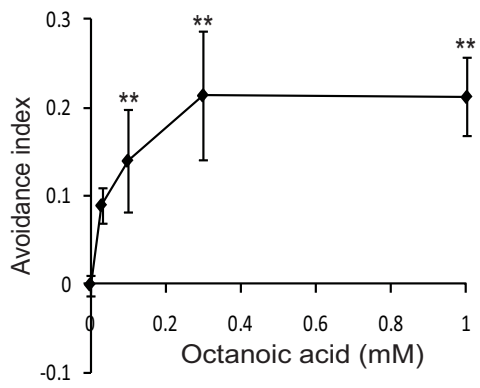

Figure 8. SiHsTRPA-activating compounds repel fire ants. $\boldsymbol{A}$, We introduced 20 fire ants at the boundary of two filter papers soaked with either compound or $10 \% \mathrm{DMSO}$, and then the ants were allowed to move for $40 \mathrm{~min}$. The number of ants in each area was counted every $5 \mathrm{~min}$ for 10-40 min, and the seven recordings were summed. In the control experiments, both filter papers were soaked with $10 \%$ DMSO. We calculated the avoidance index as shown. The avoidance index is shown at the different concentrations of $\beta$-caryophyllene $(\boldsymbol{B})$, octanoic acid $(\boldsymbol{C})$, decanoic acid $(\boldsymbol{D}), \beta$-citronellal ( $\boldsymbol{E}$; purple circle), and $\beta$-citronellol (E; blue triangle). The mean value with error bar $( \pm \mathrm{SEM} ; n=3)$ is indicated for each concentration. $* *, P$-values at $0.06,0.1$, and 0.3 mM of $\beta$-caryophyllene are $<0.0033,<0.0028$, and $<0.0007$, respectively. P-values at $0.1,0.3$, and $1 \mathrm{~mm}$ of octanoic acid $<0.009,<0.0001$, and $<0.0001$. $P$-values at $*, 0.3$, and $* *, 0.6 \mathrm{~mm}$, of decanoic acid are $<0.02$ and $<0.004$. $P$-values at $*, 0.06$, and $* *, 0.1,0.3$, and $1 \mathrm{~mm}$ of $\beta$-citronellal are $<0.02,<7.3 \times 10^{-6},<3.3 \times 10^{-6}$, and $<1.4 \times 10^{-6}$. All of above $P$-values are the results of two-tailed Dunnett test compared to $0 \mathrm{~mm}$. 
A

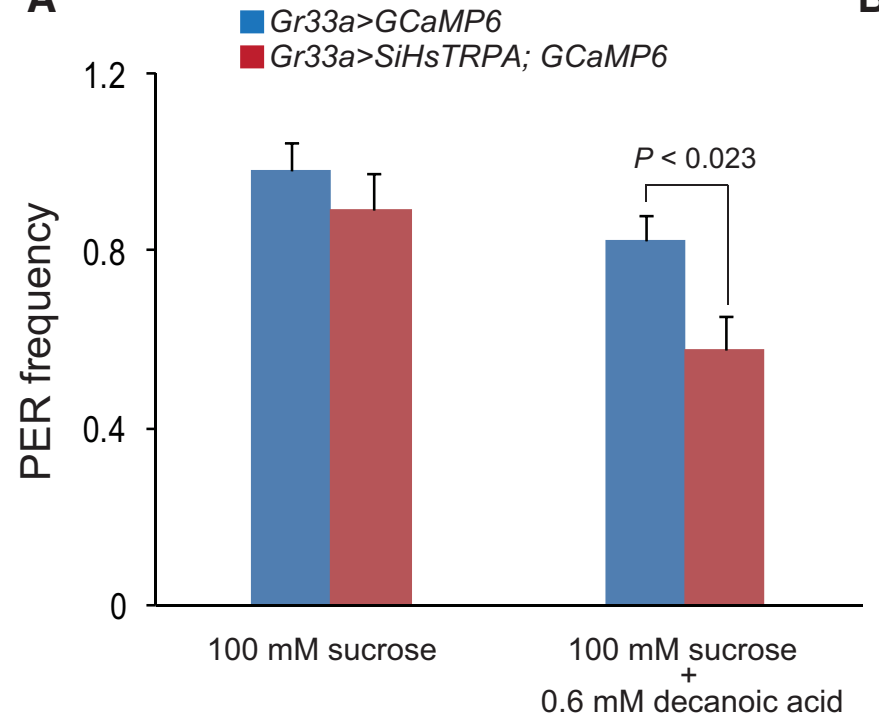

B

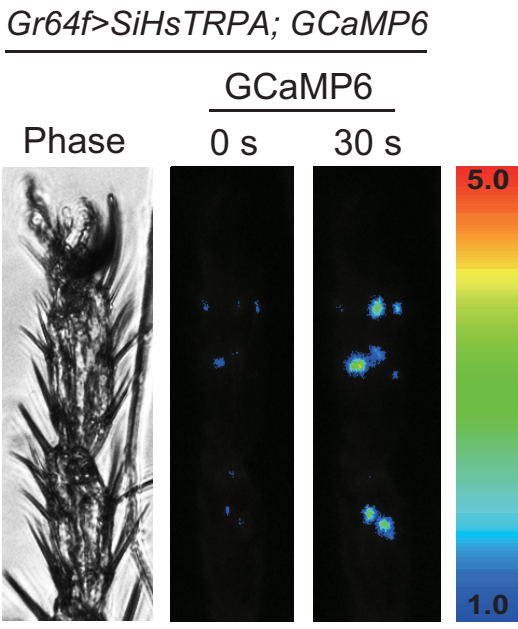

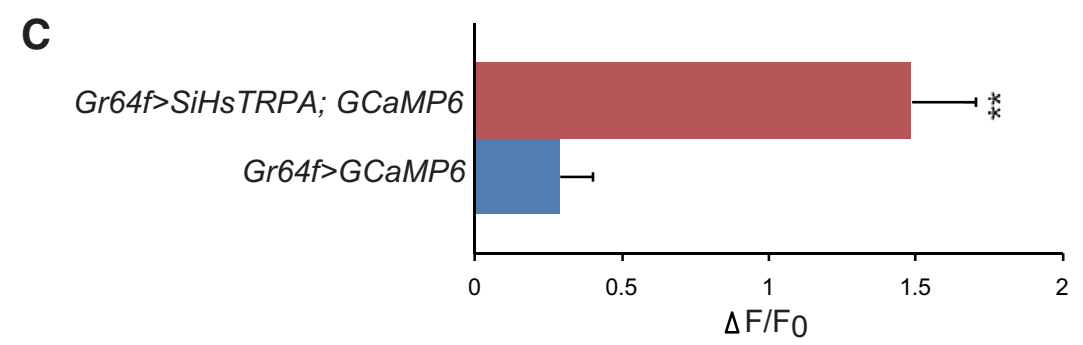

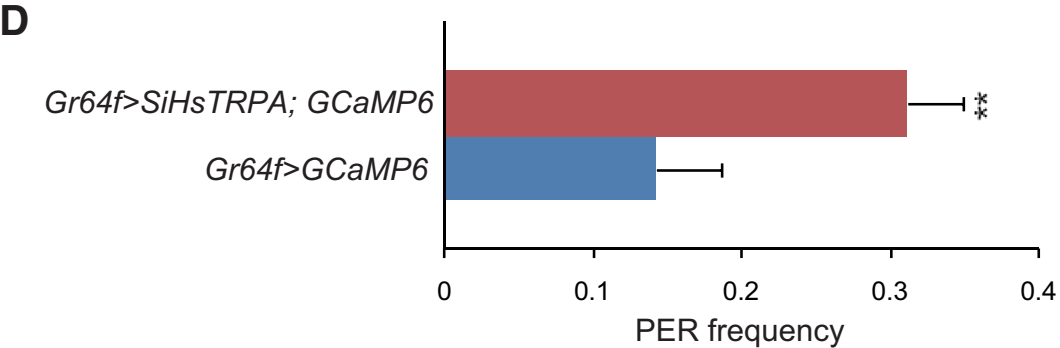

Figure 9. Decanoic acid modifies gustatory responses of $D$. melanogaster expressing SiHsTRPA. A, PER frequency of fruit flies expressing either GCaMP6 (Gr33a > GCaMP6) alone or both GCaMP6 and SiHsTRPA (Gr33a > SiHsTRPA; GCaMP6) under Gr33a-Gal4 toward $100 \mathrm{~mm}$ sucrose solution containing 0 ( $n=19-20$ for each genotype) or 0.6 mm decanoic acid $(n=19-21$ for each genotype). The mean value with error bar ( \pm SEM) is shown. PER frequency of fruit flies expressing both GCaMP6 and SiHsTRPA is significantly lower than that of those expressing GCaMP6 alone toward 100 mM sucrose containing 0.6 mm decanoic acid (two-tailed $t$ test). B, Phase and GCaMP6 images of fruit fly expressing both SiHsTRPA and GCaMP6 under Gr64f-Gal4 (Gr64f $>$ SiHsTRPA; GCaMP6) before $(0 \mathrm{~s})$ and $30 \mathrm{~s}$ after applying $0.6 \mathrm{~mm}$ decanoic acid to the distal segments of the foreleg. The increase of GCaMP6 fluorescence $(\Delta \mathrm{F})$ is indicated by pseudo-color. $\boldsymbol{A}$, Intracellular calcium changes $\left(\Delta F / F_{0}\right)$ of SiHsTRPA and GCaMP6- or GCaMP6expressing sugar taste neuron associated with 5D1 sensilla $30 \mathrm{~s}$ after applying $0.6 \mathrm{~mm}$ decanoic acid $(n=6-9)$. $* *$, Intracellular calcium level is significantly higher, with fruit flies expressing both SiHsTRPA and GCaMP6 than those expressing GCaMP6 alone (two-tailed $t$ test, $P<0.0009$ ). $\boldsymbol{D}$, PER frequency of fruit flies of Gr64f $>$ SiHsTRPA; GCaMP6 and Gr64f $>$ GCaMP6 stimulated by $0.6 \mathrm{~mm}$ decanoic acid $(n=29)$. **, Fruit flies expressing both GCaMP6 and SiHsTRPA show significantly higher PER frequency than those expressing GCaMP6 alone (two-tailed $t$ test, $P<0.0049$ ).

effect is specific to AmHsTRPA. Considering fewer domains are involved in the activation by decanoic acid/ camphor than by diallyl disulfide, we favor a hypothesis that the above protein structure may interact with both decanoic acid and camphor in AmHsTRPA, but only with decanoic acid in SiHsTRPA, and allow opening of the channel gate.

Both cinnamaldehyde and diallyl disulfide were shown to act as electrophiles to covalently modify cysteines in the $\mathrm{N}$ terminus of TRPA1 (Hinman et al., 2006; Macpher- 
son et al., 2007); however, SiHsTRPA was activated only by cinnamaldehyde, suggesting that the activation mechanisms of TRPA subfamily members by electrophiles are different. Comparing amino acid sequences of $\mathrm{AmH}-$ sTRPA and SiHsTRPA, 11 cysteines are conserved, but they also contain eight and six species-specific cysteines, respectively. Cinnamaldehyde may target the conserved cysteines, and diallyl disulfide would modify only AmHsTRPAspecific cysteine residues. However, the mechanism to activate AmHsTRPA by electrophiles cannot be simply explained by the cysteine modifications. For example, amino acids in ARs 3 and 5-7 of AmHsTRPA are essential for activation by diallyl disulfide; nevertheless, cysteine is absent in ARs 3 and 6, and ARs 5 and 7 contain only conserved cysteines. These results suggest that these ARs have important roles for channel activation, other than some of the consisting cysteines being covalently modified. SiHsTRPA has undergone amino acid substitutions in multiple domains and lost reactivity to camphor and diallyl disulfide as a result. Such amino acid substitutions changing the channel's sensitivity to chemicals may be the result of natural selection, possibly linked to the different ecological niches of these ants relative to those of bees and wasps. It is surprising that SiHsTRPA has undergone these evolutionary changes as yet by maintaining its heat sensitivity. Intriguingly, both $\beta$-citronellal (with an aldehyde group) and $\beta$-citronellol (with a hydroxyl group) can activate AmHsTRPA, but SiHsTRPA is only activated by $\beta$-citronellal. This may suggest that SiHsTRPA activation requires not only binding with $\beta$-citronellal but also the modification of an amino group by the aldehyde group.

\section{Activation of TRPA channels by fatty acids}

We found that saturated fatty acids with more than eight carbons (medium-chain fatty acids) activate both SiHsTRPA and AmHsTRPA. Because short-chain fatty acids $(C \leq 7)$ failed to activate SiHsTRPA, activation is likely to require plasma membrane penetration of a fatty acid (Kamp and Hamilton, 2006), but not extracellular protons being released from the fatty acid. Activation of human TRPA1 by octanoic acid, nonanoic acid, decanoic acid, and lauric acid was previously reported (Terada et al., 2011); however, another report showed that mouse and rat TRPA 1 channels are activated only by fatty acids with $>18$ carbons and at least three double bonds, and activation by polyunsaturated fatty acids is specific to mammalian TRPA1 channels (Motter and Ahern, 2012). The above discrepancy could be because of speciesspecific activation (primate vs. rodent TRPA1 channels). How fatty acids activate the above TRPA channels remains to be answered. A local increase in protons released from the incorporated fatty acid may activate the channel. Furthermore, the carbonyl group generated after the release of a proton may act as an electrophilic agent to covalently modify the channel. Alternatively, interaction of a fatty acid with the transmembrane segments together with the other domains may result in activation of the TRPA channel. Motter and Ahern (2012) suggested that mouse TRPA 1 is unlikely to be activated by modification of the integrity and/or fluidity of the plasma membrane by a polyunsaturated fatty acid. However, another study suggested that lipopolysaccharide activates mouse TRPA1 through mechanical alterations in the plasma membrane, since the effects are highly dependent on the structure of lipid A (Meseguer et al., 2014).

\section{Potential use of SiHsTRPA-activating compounds to control S. invicta}

Although a number of chemical and biological methods have been developed to control S. invicta in invaded areas, none of them have been very effective (for example, Collins and Callcott, 1995). Because the SiHsTRPAactivating compounds we identified are natural products that repel S. invicta, they could be used to develop such methods. These repellents would also be useful for quarantine treatment, for example, to clear $S$. invicta from imported products at a port. However, the specificity of an effect is a major issue to be addressed. All of the SiHsTRPA-activating compounds activate AmHsTRPA, and perhaps other ant HsTRPA channels, as well as arthropod TRPA1 channels (Du et al., 2015; Peng et al., 2015b; Dong et al., 2016). Screening more compounds may identify those that specifically act on SiHsTRPA. The appropriate use of such natural compounds derived from plants may serve as an alternative method to control $S$. invicta.

\section{References}

Ainsley JA, Kim MJ, Wegman LJ, Pettus JM, Johnson WA (2008) Sensory mechanisms controlling the timing of larval developmental and behavioral transitions require the Drosophila DEG/ENaC subunit, Pickpocket1. Dev Biol 322:46-55. CrossRef Medline

Appel AC, Gehret MJ, Tanley MJ (2004) Repellency and toxicity of mint oil granules to red imported fire ants (Hymenoptera: Formicidae). J Econ Entomol 97:575-580. CrossRef Medline

Bischof J, Maeda RK, Hediger M, Karch F, Basler K (2007) An optimized transgenesis system for Drosophila using germ-linespecific phi C31 integrases. Proc Natl Acad Sci U S A 104:33123317. CrossRef

Chazotte B (2011) Labeling membrane glycoproteins or glycolipids with fluorescent wheat germ agglutinin. Cold Spring Harbor Protocols 2011:pdb prot5623. CrossRef

Chen J, Cantrell CL, Duke SO, Allen ML (2008) Repellency of callicarpenal and intermedeol against workers of imported fire ants (Hymenoptera: Formicidae). J Econ Entomol 101:265-271. Medline

Chen T-W, Wardill TJ, Sun Y, Pulver SR, Renninger SL, Baohan A, Schreiter ER, Kerr RA, Orger MB, Jayaraman V, Looger LL, Svoboda K, Kim DS (2013) Ultrasensitive fluorescent proteins for imaging neuronal activity. Nature 499:295-300. CrossRef Medline

Collins H, Callcott A (1995) Effectiveness of spot insecticide treatments for red imported fire ant (Hymenoptera, Formicidae) control. J Entomol Sci 30:489-496. CrossRef

Cordero-Morales JF, Gracheva EO, Julius D (2011) Cytoplasmic ankyrin repeats of transient receptor potential A1 (TRPA1) dictate sensitivity to thermal and chemical stimuli. Proc Natl Acad Sci U S A 108:E1184-E1191. CrossRef

Damann N, Voets T, Nilius B (2008) TRPs in our senses. Curr Biol 18:R880-R889. CrossRef Medline

Dani FR, Cannoni S, Turillazzi S, Morgan ED (1996) Ant repellent effect of the sternal gland secretion of Polistes dominulus (Christ) and P-sulcifer (Zimmermann). (Hymenoptera:Vespidae). J Chem Ecol 22:37-48. CrossRef 
Dong X, Kashio M, Peng G, Wang X, Tominaga M, Kadowaki T (2016) Isoform-specific modulation of the chemical sensitivity of conserved TRPA1 channel in the major honey bee ectoparasitic mite, Tropilaelaps mercedesae. Open Biol 6:160042. CrossRef

Du EJ, Ahn TJ, Choi MS, Kwon I, Kim HW, Kwon JY, Kang K (2015) The mosquito repellent citronellal directly potentiates Drosophila TRPA1, facilitating feeding suppression. Mol Cells 38:911-917. CrossRef Medline

Dutcher JD, Beaver RW (2005) Butyl carbitol acetate as an ant repellent on the pecan tree trunk. J Entomol Sci 40:401-408. CrossRef

Elsik CG, Tayal A, Diesh CM, Unni DR, Emery ML, Nguyen HN, Hagen DE (2016) Hymenoptera Genome Database: integrating genome annotations in HymenopteraMine. Nucleic Acids Res 44: D793-D800. CrossRef Medline

Fernandez-Melendez S, Miranda A, Garcia-Gonzalez J, Barber D, Lombardero M (2007) Anaphylaxis caused by imported red fire ant stings in Malaga, Spain. J Investig Allergol Clin Immunol 17:48-49.

Fu JT, Tang L, Li WS, Wang K, Cheng DM, Zhang ZX (2015) Fumigant toxicity and repellence activity of camphor essential oil from cinnamonum camphora siebold against Solenopsis invicta workers (Hymenoptera:Formicidae). J Insect Sci 15:129CrossRef Medline

Gadau J, Helmkampf M, Nygaard S, Roux J, Simola DF, Smith CR, Suen G, Wurm Y, Smith CD (2012) The genomic impact of 100 million years of social evolution in seven ant species. Trends Genet 28:14-21. CrossRef Medline

Hamada FN, Rosenzweig M, Kang K, Pulver SR, Ghezzi A, Jegla TJ, Garrity PA (2008) An internal thermal sensor controlling temperature preference in Drosophila. Nature 454:217-255. CrossRef Medline

Hinman A, Chuang H-h, Bautista DM, Julius D (2006) TRP channel activation by reversible covalent modification. Proc Natl Acad Sci U S A 103:19564-19568. CrossRef Medline

Huang CL, Fu JT, Liu YK, Cheng DM, Zhang ZX (2015) The insecticidal and repellent activity of soil containing cinnamon leaf debris against red imported fire ant workers. Sociobiol 62:46-51. CrossRef

Jabba S, Goyal R, Sosa-Pagan JO, Moldenhauer H, Wu J, Kalmeta B, Bandell M, Latorre R, Patapoutian A, Grandl J (2014) Directionality of temperature activation in mouse TRPA1 ion channel can be inverted by single-point mutations in ankyrin repeat six. Neuron 82:1017-1031. CrossRef Medline

Jiao Y, Moon SJ, Wang X, Ren Q, Montell C (2008) Gr64f is required in combination with other gustatory receptors for sugar detection in Drosophila. Curr Biol 18:1797-1801. CrossRef Medline

Julius D (2013) TRP channels and pain. Annu Rev Cell Dev Biol 29:355-384. CrossRef Medline

Kadowaki T (2015) Evolutionary dynamics of metazoan TRP channels. Pflugers Arch 467:2043-2053. CrossRef Medline

Kafle L, Shih CJ (2013) Toxicity and repellency of compounds from clove (Syzygium aromaticum) to red imported fire ants Solenopsis invicta (Hymenoptera: Formicidae). J Econ Entomol 106:131-135. Medline

Kamp F, Hamilton JA (2006) How fatty acids of different chain length enter and leave cells by free diffusion. Prostaglandins Leukot Essent Fatty Acids 75:149-159. CrossRef Medline

Kang K, Panzano VC, Chang EC, Ni L, Dainis AM, Jenkins AM, Regna K, Muskavitch MAT, Garrity PA (2012) Modulation of TRPA1 thermal sensitivity enables sensory discrimination in Drosophila. Nature 481:76-80. CrossRef

Kim SH, Lee Y, Akitake B, Woodward OM, Guggino WB, Montell C (2010) Drosophila TRPA1 channel mediates chemical avoidance in gustatory receptor neurons. Proc Natl Acad Sci U S A 107:84408445. CrossRef Medline

Kohno K, Sokabe T, Tominaga M, Kadowaki T (2010) Honey bee thermal/chemical sensor, AmHsTRPA, reveals neofunctionalization and loss of transient receptor potential channel genes. J Neurosci 30:12219-12229. CrossRef
Kwon Y, Shim H-S, Wang X, Montell C (2008) Control of thermotactic behavior via coupling of a TRP channel to a phospholipase C signaling cascade. Nat Neurosci 11:871-873. CrossRef Medline

Lee J, Shin MK, Ryu DK, Kim S, Ryu WS (2010) Insertion and deletion mutagenesis by overlap extension PCR. Methods Mol Biol 634: 137-146. CrossRef Medline

Lee Y, Lee J, Bang S, Hyun S, Kang J, Hong ST, Bae E, Kaang BK, Kim J (2005) Pyrexia is a new thermal transient receptor potential channel endowing tolerance to high temperatures in Drosophila melanogaster. Nat Genet 37:305-310. CrossRef Medline

Liu L, Li Y, Wang R, Yin C, Dong Q, Hing H, Kim C, Welsh MJ (2007) Drosophila hygrosensation requires the TRP channels water witch and nanchung. Nature 450:294-298. CrossRef

Macpherson LJ, Dubin AE, Evans MJ, Marr F, Schultz PG, Cravatt BF, Patapoutian A (2007) Noxious compounds activate TRPA1 ion channels through covalent modification of cysteines. Nature 445: 541-545. CrossRef Medline

Matsuura H, Sokabe T, Kohno K, Tominaga M, Kadowaki T (2009) Evolutionary conservation and changes in insect TRP channels. BMC Evol Biol 9:228. CrossRef Medline

Meseguer V, Alpizar YA, Luis E, Tajada S, Denlinger B, Fajardo O, Manenschijn JA, Fernández-Peña C, Talavera A, Kichko T, Navia B, Sánchez A, Señarís R, Reeh P, Pérez-García MT, López-López JR, Voets T, Belmonte C, Talavera K, Viana F (2014) TRPA1 channels mediate acute neurogenic inflammation and pain produced by bacterial endotoxins. Nat Commun 5:3125. CrossRef Medline

Miyamoto T, Chen Y, Slone J, Amrein H (2013) Identification of a Drosophila glucose receptor using $\mathrm{Ca}^{2+}$ imaging of single chemosensory neurons. PLoS One 8:e56304. CrossRef Medline

Moon SJ, Lee Y, Jiao Y, Montell C (2009) A Drosophila gustatory receptor essential for aversive taste and inhibiting male-to-male courtship. Curr Biol 19:1623-1627. CrossRef Medline

Morrison L (2002) Long-term impacts of an arthropod-community invasion by the imported fire ant, Solenopsis invicta. Ecology 83:2337-2345. [CrossRef][10.1890/0012-9658(2002)083[2337: LTIOAA]2.0.CO;2]

Motter AL, Ahern GP (2012) TRPA1 is a polyunsaturated fatty acid sensor in mammals. PLoS One 7:e38439. CrossRef Medline

Nagel M, Kleineidam CJ (2015) Two cold-sensitive neurons within one sensillum code for different parameters of the thermal environment in the ant Camponotus rufipes. Front Behav Neurosci 9:240. CrossRef Medline

Neely GG, Keene AC, Duchek P, Chang EC, Wang Q-P, Aksoy YA, Rosenzweig M, Costigan M, Woolf CJ, Garrity PA, Penninger JM (2011) TrpA1 regulates thermal nociception in Drosophila. PLoS One 6:e24343. CrossRef Medline

Ni L, Bronk P, Chang EC, Lowell AM, Flam JO, Panzano VC, Theobald DL, Griffith LC, Garrity PA (2013) A gustatory receptor paralogue controls rapid warmth avoidance in Drosophila. Nature 500: 580-584. CrossRef Medline

Nilius B, Owsianik G (2011) The transient receptor potential family of ion channels. Genome Biol 12:218. CrossRef Medline

Ovchinnikov S, Kinch L, Park H, Liao Y, Pei J, Kim DE, Kamisetty H, Grishin NV, Baker D (2015) Large-scale determination of previously unsolved protein structures using evolutionary information. Elife 4:e09248. CrossRef Medline

Peng G, Shi X, Kadowaki T (2015a) Evolution of TRP channels inferred by their classification in diverse animal species. Mol Phylogenet Evol 84:145-157. CrossRef Medline

Peng G, Kashio M, Morimoto T, Li T, Zhu J, Tominaga M, Kadowaki $\mathrm{T}$ (2015b) Plant-derived tick repellents activate the honey bee ectoparasitic mite TRPA1. Cell Rep 12:190-202. CrossRef Medline

Ramirez-Esquivel F, Zeil J, Narendra A (2014) The antennal sensory array of the nocturnal bull ant Myrmecia pyriformis. Arthropod Struct Dev 43:543-558. CrossRef Medline

Renthal R, Velasquez D, Olmos D, Hampton J, Wergin WP (2003) Structure and distribution of antennal sensilla of the red imported fire ant. Micron 34:405-413. CrossRef Medline 
Rosenzweig M, Brennan KM, Tayler TD, Phelps PO, Patapoutian A, Garrity PA (2005) The Drosophila ortholog of vertebrate TRPA1 regulates thermotaxis. Genes Dev 19:419-424. CrossRef Medline

Ruchty M, Roces F, Kleineidam CJ (2010a) Detection of minute temperature transients by thermosensitive neurons in ants. J Neurophysiol 104:1249-1256.

Ruchty M, Helmchen F, Wehner R, Kleineidam CJ (2010b) Representation of thermal information in the antennal lobe of leaf-cutting ants. Front Behav Neurosci 4:174. CrossRef Medline

Saito S, Tominaga M (2015) Functional diversity and evolutionary dynamics of thermoTRP channels. Cell Calcium 57:214-221. CrossRef Medline

Sakulku U, Nuchuchua O, Uawongyart N, Puttipipatkhachorn S, Soottitantawat A, Ruktanonchai U (2009) Characterization and mosquito repellent activity of citronella oil nanoemulsion. Int $\mathrm{J}$ Pharm 372:105-111. CrossRef Medline

Sayeed O, Benzer S (1996) Behavioral genetics of thermosensation and hygrosensation in Drosophila. Proc Natl Acad Sci U S A 93:6079-6084. Medline

Sokabe T, Tsujiuchi S, Kadowaki T, Tominaga M (2008) Drosophila painless is a $\mathrm{Ca}^{2+}$-requiring channel activated by noxious heat. $J$ Neurosci 28:9929-9938. CrossRef Medline

Sun Y, Liu L, Ben-Shahar Y, Jacobs JS, Eberl DF, Welsh MJ (2009) TRPA channels distinguish gravity sensing from hearing in Johnston's organ. Proc Natl Acad Sci U S A 106:13606-13611. CrossRef Medline

Terada Y, Narukawa M, Watanabe T (2011) Specific hydroxy fatty acids in royal jelly activate TRPA1. J Agric Food Chem 59:26272635. CrossRef Medline

Tracey WD, Wilson RI, Laurent G, Benzer S (2003) Painless, a Drosophila gene essential for nociception. Cell 113:261-273. Medline
Venkatachalam K, Montell C (2007) TRP channels. Ann Rev Biochem 76:387-417. CrossRef Medline

Vinson S (2013) Impact of the invasion of the imported fire ant. Insect Sci 20:439-455. CrossRef Medline

Wheeler GS, Massey LM, Southwell IA (2003) Dietary influences on terpenoids sequestered by the biological control agent Oxyops vitiosa: effect of plant volatiles from different Melaleuca quinquenervia chemotypes and laboratory host species. J Chem Ecol 29: 189-208. Medline

Wilder S, Barnum T, Holway D, Suarez A, Eubanks M (2013) Introduced fire ants can exclude native ants from critical mutualistprovided resources. Oecologia 172:197-205. CrossRef Medline

Wolfgang W, Simoni A, Gentile C, Stanewsky R (2013) The Pyrexia transient receptor potential channel mediates circadian clock synchronization to low temperature cycles in Drosophila melanogaster. Proc Biol Sci 280:20130959. CrossRef Medline

Wu D, Zeng L, Lu Y, Xu Y (2014) Effects of Solenopsis invicta (Hymenoptera: Formicidae) and its interaction with aphids on the seed productions of mungbean and rapeseed plants. $J$ Econ Entomol 107:1758-1764. CrossRef

Wurm Y, et al. (2011) The genome of the fire ant Solenopsis invicta. Proc Natl Acad Sci U S A 108:5679-5684. CrossRef Medline

Zhang N, Tang L, Hu W, Wang K, Zhou Y, Li H, Huang C, Chun J, Zhang $Z$ (2014) Insecticidal, fumigant, and repellent activities of sweet wormwood oil and its individual components against red imported fire ant workers (Hymenoptera: Formicidae). J Insect Sci 14:241. CrossRef

Zhong L, Bellemer A, Yan H, Honjo K, Robertson J, Hwang RY, Pitt GS, Tracey WD (2012) Thermosensory and nonthermosensory isoforms of Drosophila melanogaster TRPA1 reveal heat-sensor domains of a thermoTRP channel. Cell Rep 1:43-55. CrossRef 